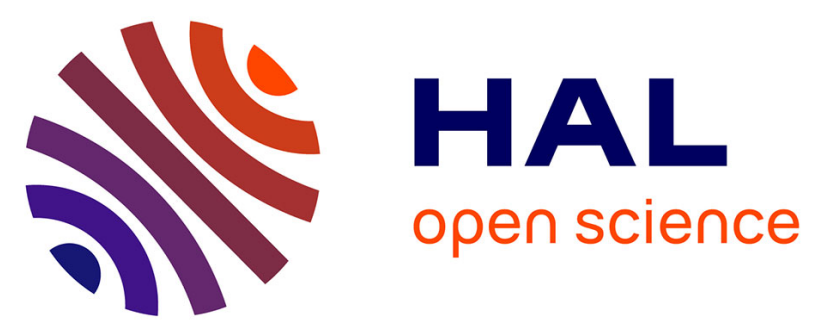

\title{
A complete NCI perspective: from new bonds to reactivity
}

\author{
Christophe Narth, Zeina Maroun, Roberto A. Boto, Robin Chaudret, \\ Marie-Laure Bonnet, Jean-Philip Piquemal, Julia Contreras-García
}

\section{- To cite this version:}

Christophe Narth, Zeina Maroun, Roberto A. Boto, Robin Chaudret, Marie-Laure Bonnet, et al.. A complete NCI perspective: from new bonds to reactivity. Esmail Alikhani, Remi Chauvin, Christine Lepetit, Bernard Silvi. Applications of Topological Methods in Molecular Chemistry, 22, Springer, pp.491-527, 2016, Challenges and Advances in Computational Chemistry and Physics 10.1007/978-3319-29022-5_18. hal-01224203

\section{HAL Id: hal-01224203 \\ https://hal.science/hal-01224203}

Submitted on 10 Nov 2015

HAL is a multi-disciplinary open access archive for the deposit and dissemination of scientific research documents, whether they are published or not. The documents may come from teaching and research institutions in France or abroad, or from public or private research centers.
L'archive ouverte pluridisciplinaire HAL, est destinée au dépôt et à la diffusion de documents scientifiques de niveau recherche, publiés ou non, émanant des établissements d'enseignement et de recherche français ou étrangers, des laboratoires publics ou privés. 


\title{
A complete NCI perspective: from new bonds to reactivity
}

Christophe Narth, Zeina Maroun, Roberto A. Boto, Robin Chaudret, Marie-Laure

Bonnet, Jean-Philip Piquemal and Julia Contreras-García

\begin{abstract}
The Non-Covalent Interaction (NCI) index is a new topological tool that has recently been added to the theoretical chemist's arsenal. NCI fills a gap that existed within topological methods for the visualization of non-covalent interactions. Based on the electron density and its derivatives, it is able to reveal both attractive and repulsive interactions in the shape of isosurfaces, whose color code reveals the
\end{abstract}

Christophe Narth

UPMC Univ Paris 06, UMR 7616, Laboratoire de Chimie Théorique, F-75005, Paris,France,

CNRS, UMR 7616, Laboratoire de Chimie Théorique, F. 75005, Paris, France, e-mail: christophe.narth@1ct.jussieu.fr

Zeina Maroun

Department of Chemistry, University of Copenhagen, Universitetsparken 5, DK-2100 Copenhagen, Denmark, e-mail: zeinamaroun89@gmail.com

Roberto A. Boto

UPMC Univ Paris 06, UMR 7616, Laboratoire de Chimie Théorique, F-75005, Paris,France,

CNRS, UMR 7616, Laboratoire de Chimie Théorique, F. 75005, Paris, France, e-mail: alboto@1ct.jussieu.fr

Robin Chaudret

Scienomics, 16 rue de l'Arcade, F. 75008, Paris, France, e-mail: Robin.Chaudret@ scienomics.com

Marie-Laure Bonnet

ENSCP, Ecole Nationale Superieure de Chimie de Paris Chimie ParisTech, UMR 8247 Institut de Recherche de Chimie Paris, F-75231, Paris, France,

CNRS, UMR 8247, Institut de Recherche de Chimie Paris, F-75231, Paris, France, e-mail: marielaure.bonnet@chimie-paristech.fr

Jean-Philip Piquemal

UPMC Univ Paris 06, UMR 7616, Laboratoire de Chimie Théorique, F-75005, Paris,France,

CNRS, UMR 7616, Laboratoire de Chimie Théorique, F. 75005, Paris, France,e-mail: jpp@1ct.jussieu.fr

Julia Contreras-García

UPMC Univ Paris 06, UMR 7616, Laboratoire de Chimie Théorique, F-75005, Paris,France, CNRS, UMR 7616, Laboratoire de Chimie Théorique, F. 75005, Paris, France, e-mail: contrera@1ct.jussieu.fr 
nature of the interaction. It is interesting to note that NCI can even be calculated at the promolecular level, making it a suitable tool for big systems, such as proteins or DNA. Within this chapter we will review the main characteristics of NCI, its similarities with and differences from previous approaches. Special attention will be paid to the visualization of new interaction types. Being based on the electron density, NCI is not only very stable with respect to the calculation method, but it is also a suitable tool for detecting new bonding mechanisms, since all such mechanisms should have a detectable effect on the electron density. This type of approach overcomes the limitations of bond definition, revealing all interaction types, irrespective of whether they have a name or have previously been identified. Finally, we will show how this tool can be used to understand chemical change along a chemical reaction. We will show several examples of torquoselectivity and put forward an explanation of selectivity based on secondary interactions which is complementary to the historical orbital approach. 


\section{Introduction}

As defined by Linus Pauling, "Chemistry is the science of substances: their structure, their properties, and the reactions that change them into other substances". [1] The first aspects, structure and properties, are clearly associated with the arrangement of atoms in a molecule, i.e. the chemical bond. These bonds determine Pauling's third aspect, chemical reactivity. In other words, chemical bonds are the undisputed foundation of chemistry and their visualization should allow chemists to understand how molecules behave at the most fundamental level.

Achievement of a mechanistic understanding of chemical and biological functions as well as the structure of solid materials depends on knowing the geometric structure and the nature of bonds. But, despite the "chemical bond being a fundamental concept in chemistry, "what is a chemical bond?" still remains a critical question for the chemical community because of the lack of a unique definition as well as an unclear understanding of its physical nature.

Successful numerical solution of the Schrödinger equation has yielded energies and properties of atoms and molecules, but not yet a clear physical explanation of chemical bonding. There is even a controversy on the mechanistic origin of the most "simple" chemical bond, covalent bonding, as it was remarked by Burdett in his classical book. [2]

As recently as in 2007, a special issue was devoted to the "90 Years of Chemical Bonding". [3] In this issue, the chemical bond was compared to a unicorn, "a mythical but useful creature, which brings law and order $[\cdots]$ in an otherwise chaotic and disordered world". [4] Everyone knows how it looks despite nobody ever having seen one. [4-6] This line of reasoning is similar to Coulson's comment: "Sometimes it seems to me that a bond between two atoms has become so real, so tangible, so friendly, that I can almost see it. Then I awake with a little shock, for a chemical bond is not a real thing. It does not exist. No one has ever seen one. No one ever can. It is a figment of our own imagination." [7] Even chemical bonds have been described as "noumena" rather than "phenomena". [8-10]

Chemical bonds together with other concepts such as atomic orbitals, electron shells, lone pairs, aromaticity, atomic charges, (hyper-) conjugation, strain, etc. do not correspond to physical observables. Such concepts therefore cannot be unambiguously defined in pure quantum theory, but constitute a rich set of "fuzzy", yet invaluably useful concepts. [11-14] They lead to constructive ideas and developments when appropriately used and defined.

In chemistry as well as in physics, advanced theories are held by two milestones: i) a mathematical structure/formalism disclosing the basic entities of the theory and their mathematical relationships, and ii) an "interpretative" recipe of basic entities of the theory. The latter discloses the qualitative meaning of the basic entities and their relation to other known entities within and beyond the theory. It is important to highlight that the connection between the mathematical formalism and its interpretation is always subtle. This problem can be traced back to the lack of a clear and unambiguous definition of a bond in quantum mechanics and the plethora of inter- 
pretations that have been introduced with various "meanings" of the "mathematical symbols/entities" of the theory. [15]

In front of this quandary, two opposite attitudes can be envisaged. On the one hand, the old and negative statement of the French mathemathician R. Thom: "Il me faut cependant avouer que la chimie proprement dite ne m'a jamais beaucoup intéressée. Pourquoi? Peut-être parce que des notions telles que celles de valence, de liaison chimique etc., m'ont toujours semblé peu claires du point de vue conceptuel." (I should admit that chemistry never really interested me. Why? Perhaps because notions such as those of valence, chemical bond, etc., always appeared unclear to me from the conceptual point of view). On the other hand, the more actual and pragmatic comment of Alvarez et al.: "Chemistry has done more than well in a universe of structure and function on the molecular level with just this imperfectly defined concept of a chemical bond. Or maybe it has done so well precisely because the concept is flexible and fuzzy". [16]

However, it is important to note that scientific arguments, debates, and controversies are at the heart of chemistry. This situation has been clearly stated in the very recent paper entitled "The Nature of the Fourth Bond in the Ground State of $\mathrm{C}_{2}$ : The Quadruple Bond Conundrum" by Danovich et al., [17] in which these authors recongnize that they are in front of a "Rashomon effect", in which the bonding picture is becoming too fuzzy to be constructive anymore.

In trying to overcome this dichotomy, topological analysis has become one of the most useful tools to characterize interactions. Visualization of bonding interactions between atoms and molecules is a long-standing quest in theoretical and computational chemistry. The main interest lies in creating a tool that enables not only to see the interaction, but also to interpret its character and properties. Different types of bonding can be revealed by various topological methods, each based on different scalar fields. [18] This chapter deals with a new interpretative tool, NCI (standing for Non-Covalent Interactions), for revealing non-covalent interactions, which tries to brind some intuitive order into this fuzzy set of ideas.

\subsection{Historical framework}

Over the years, different approaches have been developed to reveal chemical bonds. Covalent bonds are intuitively represented using conventional Lewis structures. [19] Molecular Orbital (MO) theory has been very useful and successful for the theoretical analysis of chemical reactions and chemical reactivity. The frontier orbital theory [20] and the orbital symmetry rules of Woodward and Hoffman [21] are paradigmatic examples of the possibilities of quantum chemistry within the MO theory.

To reduce the dimensionality of the problem, three-dimensional interpretative approaches have been introduced. The conceptual density functional theory pioneered by Parr et al. [22] has been at the origin of very useful reactivity descriptors. Another low dimensional approach has originally been developed by Bader: [23-25] 
the topological approach. Within these approaches, 3D space is divided following the gradient of a scalar function into mutually disjointed regions. Bader's QTAIM (Quantum Theory of Atoms In Molecules) theory is based on the topological analysis of the density and provides an atomic picture of the system. Chemical bonds and the underlying molecular graph may be traced by the analysis of its bond critical points (BCPs, first order saddle points). Following the same philosophy, ELF [26,27] (Electron Localization Function) topological analysis divides the space into chemically intuitive regions associated with electron pairs so that electronic shells, bonds, and lone pairs are revealed. Also, purely electrostatic interactions can be analyzed using electrostatic potential maps. [28]

\subsection{Weak interactions}

Chemical interactions between a protein and a drug, or a catalyst and its substrate, self-assembly of nanomaterials, [29,30], and even some chemical reactions, [31, 32] are dominated by non-covalent interactions. This class of interactions spans a wide range of binding energies, and encompasses hydrogen bonding, dipole-dipole interactions and London dispersion [33] as well as more up to date interactions such as halogen bonds, $\mathrm{CH} \cdots \pi$ and $\pi \cdots \pi$ interactions. Repulsive interactions, also known as steric clashes, should not be disregarded either.

More specifically, non-covalent interactions are of paramount importance in chemistry and especially in bio-disciplines, [34,35] since they set up the force field scenario through which chemical species interact with each other without a significant electron sharing between them. They represent, in fact, the machinery through which molecules recognize themselves and establish how molecules will approach and eventually pack together.

During the last decade, non-covalent interactions have also raised a great deal of interest in the context of self-assembly [36] and crystallization, [37] whose underlying general rules are at the moment too faraway to be fully rationalized and understood. [38] Knowledge of such rules would in principle allow to build from scratch (even complex) materials exhibiting the desired properties. [29, 39, 40] Although it can not be ignored that a given observed structure is generally the outcome of a "drawing" among a plethora of energetically similar, but structurally dissimilar options, [41] understanding intermolecular non-covalent interactions and their mutual interplay in the supramolecular assemblies is nonetheless a fundamental step in making progress in structural prediction and evolution.

\subsubsection{Weak interactions: the need for a new approach}

Non-covalent interactions are frequently visualized using distance-dependent contacts, generally without consideration of hydrogen atoms. [42-44] Hydrogen-bonds can be identified from the molecular geometry [45] and from ELF, [46] while grid- 
based calculations from classical force fields are used to model other van der Waals interactions. [47] The crucial role of weak interactions can also be analyzed in an indirect manner through property computations (from population to electrostatic moments). [48] However, these fluctuations are not easily visualized. In other words, a visual quantum chemical approach was conspicuously missing in this scenario.

Let's look at an example to clearly pinpoint the state of the art of topological approaches to weak interactions. The image provided by QTAIM and ELF of benzene dimer is provided in Table 1. Let's first focus on a given benzene molecule: The electron density shows maxima (cusps) for the $\mathrm{C}$ and $\mathrm{H}$ atoms, whereas $\mathrm{C}-\mathrm{H}$ and $\mathrm{C}-\mathrm{C}$ bonds are represented by BCPs (in red). ELF, instead, provides a picture based on electron localization, so that isosurfaces appear around atoms and C-H bonds. In both cases the chemical structure is revealed as expected from chemical intuition. However, the ELF picture is obviously more intuitive thanks to the isosurfaces.

Now, if we take a look at the complete system, the stacking dimer, we can see that intermolecular BCPs appear along with a ring critical point (second order saddle point, in yellow). The fact that interactions are related to saddle points, locates them on the interatomic surfaces, so that they highlight interatomic contact, but they do not have an associated region within this approach. Moreover, the critical points unite pairs of $\mathrm{C}$ atoms, which is not the chemical picture we have of a stacking interaction: it should appear as a benzene to benzene interaction. The VSEPR regions of benzene dimer are clearly identified by ELF, but nothing is seen for the inter-benzene stacking interaction.

In other words, both approaches fail to correctly provide a picture of delocalized interactions. Thus, it is the aim of this chapter to introduce such a tool, show its advantages over previous theories and its ability to provide a complete and holistic vision of non-covalent interactions and their change directly from the electron density. 
Table 1 Comparison between QTAIM and ELF topologies in benzene dimer

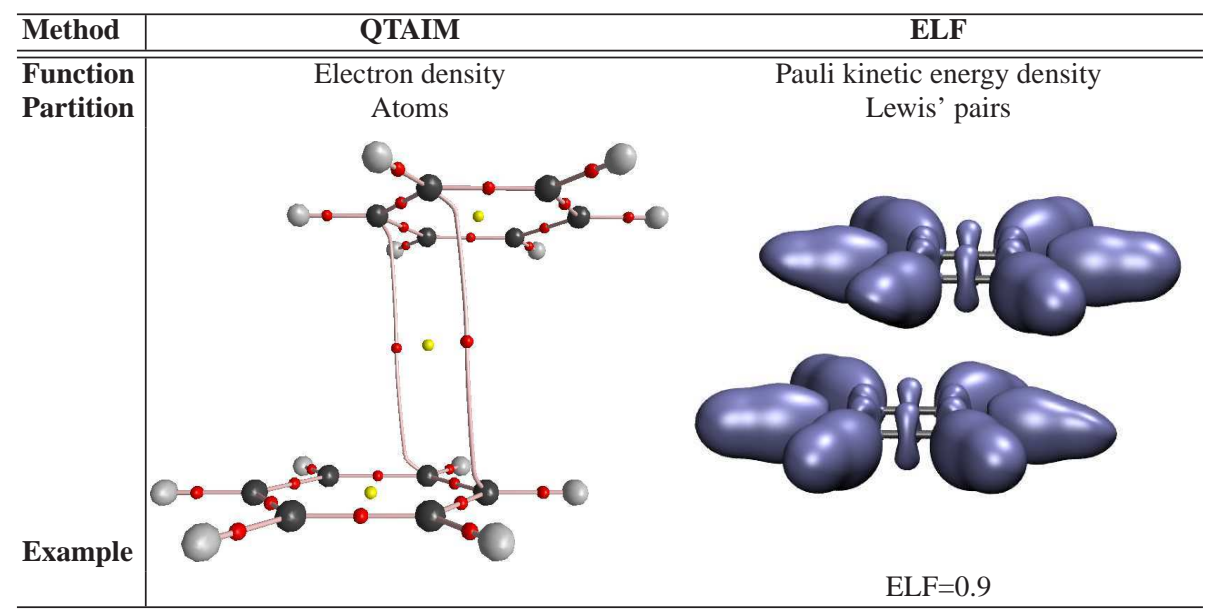




\section{NCI: Non-Covalent Interaction index}

The electron density has a fundamental advantage over MO-based descriptors because it is an experimentally accessible scalar field and it is a local function defined within the exact many-body theory, also supported by the Hohenberg-Kohn theorem. [49] The relationship between electron density topology and physical/chemical properties can be understood from the Hohenberg-Kohn theorem, which asserts that a system's ground-state properties are a consequence of its electron density. Furthermore since chemical reactions proceed by $\rho(\mathbf{r})$ redistributions, methods that analyze $\rho(\mathbf{r})$ distributions should help to understand the electron structure of molecules and thus chemical reactivity (see Section 8).

Our approach, introduced in the coming sections, uses the density and its derivatives, allowing simultaneous analysis and visualization of all non-covalent interaction types as real-space surfaces, thus adding an important tool to the chemist's arsenal. [50-52]

\subsection{The reduced density gradient}

The reduced density gradient, $s$ or RDG, is a fundamental dimensionless quantity in DFT used to describe the deviation from a homogeneous electron distribution. $[49,53,54]$ Properties of the reduced gradient have been investigated in depth in the process of developing increasingly accurate functionals. [55]

The origin of the reduced density gradient can be traced to the generalized gradient contribution to the GGA exchange energy, $E_{X}^{G G A}$, from density-functional theory:

$$
E_{X}^{G G A}-E_{X}^{L D A}=-\sum \int F(s) \rho^{4 / 3}(\mathbf{r}) d \mathbf{r}
$$

where $F(s)$ is a function of $s$ for a given spin with

$$
s=\frac{1}{C_{F}} \frac{|\nabla \rho|}{\rho^{4 / 3}},
$$

$c_{F}$ being the Fermi constant, $c_{F}=2\left(3 \pi^{2}\right)^{1 / 3}$ and the $4 / 3$ exponent of the density ensuring that $s$ is a dimensionless quantity.

$s$ accounts for local density inhomogeneities due to its differential behavior depending on the chemical region of the molecule. The reduced density gradient assumes large values in the exponentially-decaying density tails far from the nuclei, where the density denominator approaches zero more rapidly than the gradient numerator. Small values of $s$ occur close to the nuclei, due to the combination of large densities and small density gradients. The lower bound on the reduced density gradient is zero, as occurs throughout a homogeneous electron gas and at bond critical points. 
The effect of bonding on the reduced density gradient is especially easy to visualize when $s$ is plotted as a function of the density. Graphs of $s(\rho)$ assume the form $f(x)=a x^{-1 / 3}$, where $a$ is a constant (see Appendix I). This can easily be proven from a STO model density. For a single atomic orbital $\psi=e^{-\alpha r}$, the density is $\rho=e^{-2 \alpha r}$ and the gradient is $\nabla \rho=-2 \alpha \rho$, such that

$$
s(\rho)=\frac{1}{C_{F}} \frac{2 \alpha \rho}{\rho^{4 / 3}}=\frac{2 \alpha}{C_{F}} \rho^{-1 / 3} .
$$

When there is overlap between atomic orbitals, a spike in the $s(\rho)$ diagram appears (Figure 1). The points forming this spike can identify the interaction when they are mapped back to real space. This procedure is able to reveal non-covalent interactions.

To explore the features associated with small reduced gradients, we first examine plots of $s$ versus $\rho$ (Figure 1). These plots were generated by evaluating the B3LYP $[56,57]$ density and reduced gradients on cuboid grids, with a 0.1 au step size, for each molecule or dimer. To provide even more sampling of the small low-density, low-gradient regions in hydrogen-bonded complexes, additional calculations were performed for water and formic acid dimers with a much denser 0.025 au grid.

a

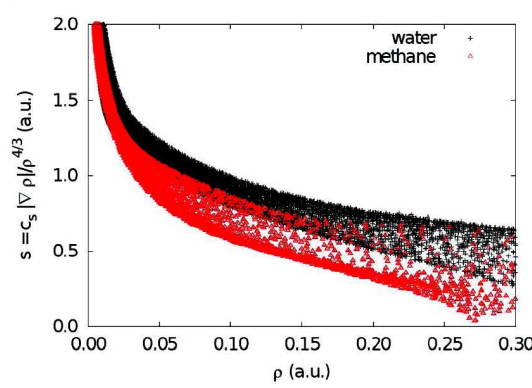

c

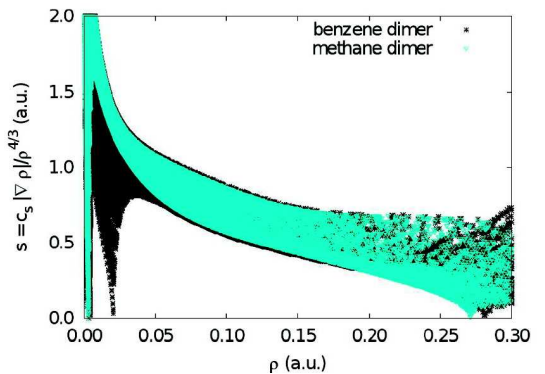

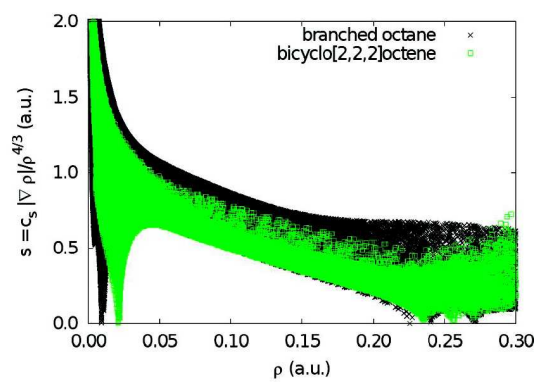

d

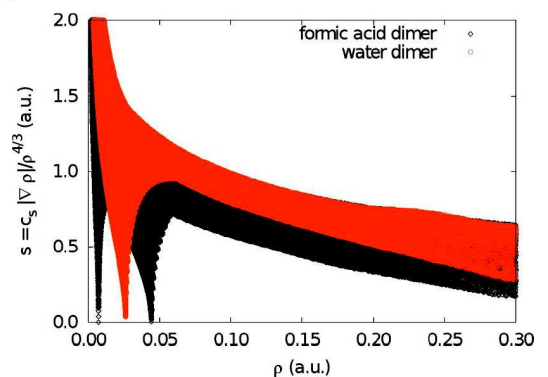

Fig. 1 Plots of the electron density and its reduced gradient for methane, water, branched octane, bicyclo $[2,2,2]$ octene, and the homomolecular dimers of methane, benzene, water, and formic acid. The data was obtained by evaluating B3LYP/6-31G* density and gradient values on cuboid grids. Reprinted with permission from Ref. [50]. Copyright 2010 American Chemical Society. 
Plotting $s$ versus $\rho$, as in Figure 1, reveals the basic pattern of intramolecular interactions. Methane (Figure 1a) illustrates the typical covalent bond pattern. The top left-side points (small density and large reduced gradient) correspond to the exponentially-decaying tail regions of the density, far from the nuclei. The points on the bottom right side (density values of ca. 0.25 au and low reduced gradient) correspond to the $\mathrm{C}-\mathrm{H}$ covalent bonds. Covalent bonds have their characteristic BCP in the electron density, corresponding to $s=0$. Regions near the nuclei have larger density values and appear beyond the right edge of the plot. The plot has an overall shape of the form $a \rho^{-1 / 3}$ because atomic and molecular densities are piecewise exponential. The results for water are very similar, the only difference being that the covalent bonds lie at higher density values, past the edge of the plot. In Figure 1b-d, we consider six examples of chemical systems displaying various types of non-covalent interactions. Plots of $s$ versus $\rho$ for these systems all exhibit a new feature: one or more spikes in the low-density, low-gradient region, a signature of non-covalent interactions. This is the basis of NCI.

\subsection{The density second eigenvalue}

According to the divergence theorem, [58] the sign of the Laplacian of the density, $\nabla^{2} \rho$, indicates whether the net gradient flux is entering, $\nabla^{2} \rho<0$, or leaving, $\nabla^{2} \rho>0$, an infinitesimal volume around a reference point. Hence, it highlights whether the density is concentrated or depleted at that point, relative to the surrounding environment. To differentiate between different types of weak interactions one cannot resort to the sign of the Laplacian itself (as is common within AIM theory) since it is dominated by the principle axis of variation and is positive for all closed-shell interactions. [59]

To understand bonding in more detail, it is often useful to decompose the Laplacian into the contributions along the three particular axes of maximal variation. These components are the three eigenvalues $\lambda_{i}$ of the electron-density Hessian matrix, such that, $\nabla^{2} \rho=\lambda_{1}+\lambda_{2}+\lambda_{3},\left(\lambda_{1}<\lambda_{2}<\lambda_{3}\right)$. At points with zero gradient, analysis of the Hessian eigenvalues is analogous to determining the signature of the critical point. Thus, at nuclei (cusps/maxima of $\rho$ ), all the eigenvalues are negative, while at the center of cages or holes (minima of $\rho$ ) all the eigenvalues are positive. In the remaining points of space $\lambda_{3}>0, \lambda_{1}<0$, and $\lambda_{2}$ can be either positive or negative. Within the NCI framework, the sign of $\lambda_{2}$ (i.e. the perpendicular plane) enables identification of the interaction type. Attractive interactions appear at $\lambda_{2}<0$ whereas in the cases where $\lambda_{2}$ is positive (as in rings or cages), usually several atoms interact but are not bonded, which corresponds to steric crowding according to classical chemistry.

Both van der Waals interactions and hydrogen bonds show negatives value of $\lambda_{2}$ at the critical point (with $\lambda_{2} \simeq 0$ for van der Waals interactions). This can be attributed to the homomorphic virial path associated with the bonding direction, which defines a line along which the potential-energy density is maximally negative. Con- 
versely, non-bonding interactions, such as steric crowding, result in density depletion, such that $\lambda_{2}>0$. Analogously, the homeomophism ensures that these critical points (both ring and cages points) identify lines of minimally-negative potentialenergy density.

\section{Interpreting NCI}

\subsection{The 2D plot}

Once the second eigenvalue has been introduced to separate attractive from repulsive interactions, it is necessary to categorize the interactions by their strength. This can be done thanks to the properties of the corresponding critical points. Characteristic densities of van der Waals interactions are much smaller than densities at which hydrogen bonds appear. However, steric clashes and hydrogen bonds span similar density ranges and overlap in plots of $s(\rho)$. This is illustrated for the phenol dimer in Figures 2a-b. This is a hydrogen-bonded complex that exhibits non-bonding interactions within each benzene ring and a stacking interaction between the benzene rings. We thus have the three main types of interactions: Van der Waals, HB and steric clashes. Whereas van der Waals is well differenciated because it appears at smaller densities, the steric clash and the hydrogen bond spikes overlap in Figure $2 \mathrm{a}$.

The interaction type can be distinguished if the $s(\rho)$ diagrams are modified by plotting $\operatorname{sign}\left(\lambda_{2}\right) \rho$ as the ordinate. Analysis of the sign of $\lambda_{2}$ thus helps to discern between the different types of weak interactions, whereas the density itself provides information about their strength; both are combined in the value of $\operatorname{sign}\left(\lambda_{2}\right) \rho$. When the Hessian eigenvalues are considered, the different nature of these interactions is made clear: the benzene-ring interactions remain at positive values, whereas the hydrogen bond now lies at negative values, within the attractive regime. The NCI spikes nearest zero density correspond to weakly-attractive dispersion interactions between the phenyl rings.

\section{$3.23 \mathrm{D}$ plot}

\subsubsection{D visualization}

The 3D spatial visualisation of the non-covalent interactions as defined above is done using the data from the 2D plots as input to construct 3D plots composed of reduced density gradient isosurfaces.

In a nutshell, a cut-off value of $s$ close to zero, typically $s<0.5$, is chosen in order to recover all the non-covalent interactions in the system, i.e. all the spikes 

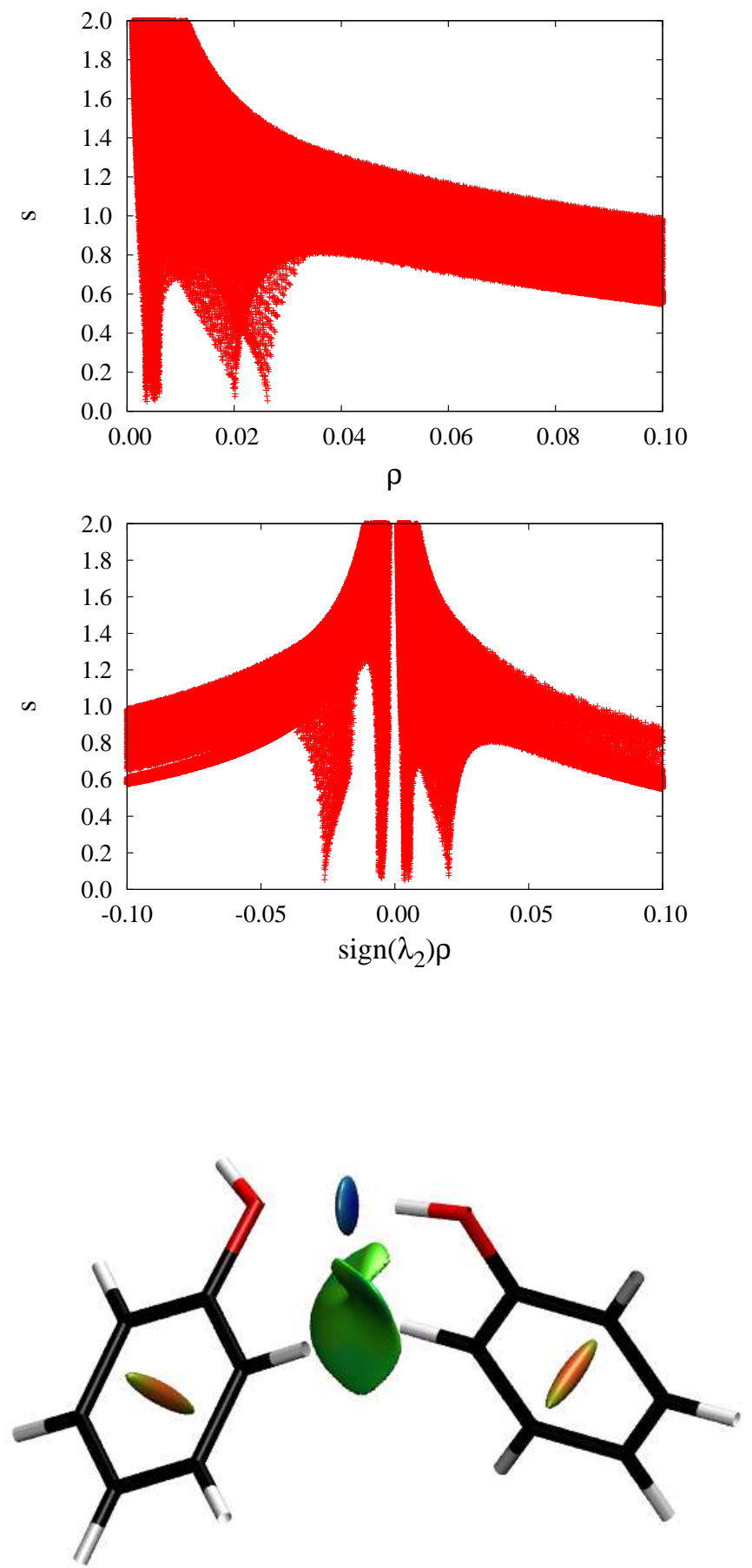

Fig. 2 Plots of (a) $s(\rho)$, (b) NCI isosurface, for the phenol dimer. The $s=0.6$ isosurface is colored over the range $-0.03<\operatorname{sign}\left(\lambda_{2}\right) \rho<+0.03$ au. 
of the $2 \mathrm{D}$ plots. The corresponding reduced density gradient isosurfaces give rise to closed domains in the molecular space which highlight the spatial localization of the interactions within the system (see Figure 2c). Since 3D isosurfaces are, by definition, regions of low reduced gradient, the density is nearly constant within these.

At this stage, however, the types of interaction corresponding to the several isosurfaces are not apparent. In order to discriminate between them, the density oriented by the sign of $\lambda_{2}$ is further used (as in the 2D plot). A RGB (red-green-blue) coloring scheme is chosen to rank interactions, where red is used for destabilizing interactions, blue for stabilizing interactions and green for delocalized weak interactions. The intensity of these colors (i.e. the deepness of the color) is associated with a higher local density and therefore to a stronger interaction.

The isovalue (or cut-off) of $\mathrm{s}(\rho)$ chosen for plotting the 3D isosurface determines which features will appear in the NCI plot as well as their spatial extension. On the one hand, all NCI spikes do not strictly achieve $s=0$, so that too low a value might miss some of the interactions of interest. [60] On the other hand, too high a value would disclose atomic tails of the density. [61] The cut-off is therefore chosen from the 2D plot so that all spikes, but only spikes, are captured to render a meaningful picture which recovers both attractive and repulsive interactions.

\subsubsection{D and 3D shapes}

Topological features of the electron density are very stable with respect to the calculation method. The main effect of different methods on the $s$ vs. $\rho$ diagram is a shift of spikes. The only rule of thumb seems that the same $s$ value should be used when comparing to each other the various NCI, both in the same or in different systems, provided a single method was employed to obtain the various electron densities. This not being the case, different $s$ values are seemingly required to compare on similar grounds the $s$-based results for differently computed electron density (e.g. from wavefunction, multipolar). In other words, a shift of cut-offs is needed to obtain comparable images This can be related to the fact that $s$ roughly behaves like

$\rho^{-1 / 3}$ (see Eq. 3), so that the effect of the method on the density is directly followed by the $s$-value. This information is crucial when moving towards bigger systems (see Section 6).

It emerges that a one-to-one inverse correlation seems to exist among the directionality (and the strength) of specific non-covalent interactions and the surface/volume ratio of the corresponding $s$ isosurface. In particular, the stronger the interaction, the smaller and more disc-shaped the $s$ surface appears in real space (and the more negative the $\rho \operatorname{sign}\left(\lambda_{2}\right)$ values are).

In some cases, noticeably in ring closings, bicolored isosurfaces appear (see Figure 3). They result from stabilizing features (revealed by the blue color), counterbalanced by destabilising interactions due to steric crowding (revealed by the red color), such as ring closure. [62] 


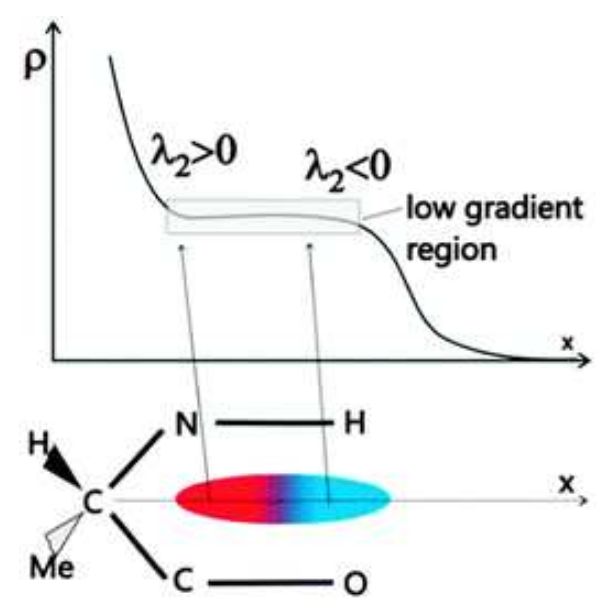

Fig. 3 Scheme of a mixed color isosurface in a C5 ring formed in the groove between a carbonyl and a $\mathrm{NH}$ group within a peptide residue. Within this isosurface, the curvature varies sign, leading to two well differenciated parts. A blue external part which illustrates the directional $\mathrm{NH} \cdots \mathrm{O}$ interaction and a red part which is indicative of the strain in the 5-membered ring resulting from a multicentric density in the inner region close to the backbone. In the top panel the variation of the density and the sign of $\lambda_{2}$ is depicted. Reproduced from Ref. [62] with permission from the PCCP Owner Societies.

\section{Small molecules}

To explore the features associated with small reduced gradients, we first examine 3D NCI plots in representative small molecules.

Figure 4 displays NCI isosurfaces for branched octane, bicyclo[2,2,2]octene, and the homomolecular dimers of methane, benzene, water, and formic acid. These isosurfaces provide a rich visualization of non-covalent interactions as broad areas of real space, rather than simple pair-wise contacts between atoms. We first consider the sterically-crowded molecules bicyclo[2,2,2] octene (Figure 4a) and the branched octane isomer (Figure 4b). In the first case, the low-density, low-gradient region corresponds to the center of the cage, where steric repulsion between the bridgehead carbons is expected. For the branched octane isomer, the isosurface lies between the closely-interacting methyl groups on opposite sides of the central C-C bond. The interactions are repulsive nearer the $\mathrm{C}-\mathrm{C}$ bond and weakly attractive between the hydrogen atoms. Dispersion and hydrogen bonding can also be clearly detected. In the dispersion-bound methane dimer (Figure 4d), the isosurface forms a disc between the individual monomers. For the water dimer (Figure 4e), the isosurface lies between a hydrogen donor and oxygen acceptor, characteristic of hydrogen bonds. The formic acid dimer (Figure 4f) reveals stronger HBs than in the water dimer, and also weak van der Waals interactions between the two closely-interacting acidic hydrogens. 


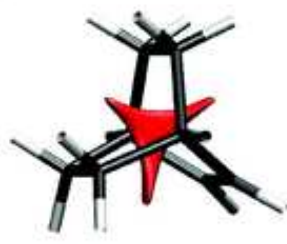

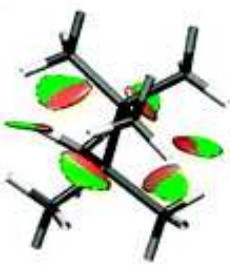

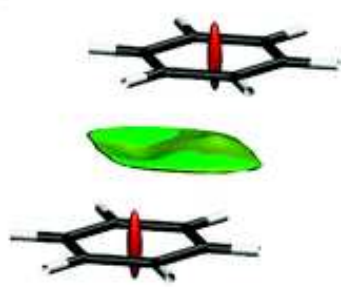

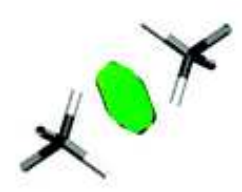

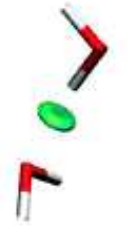

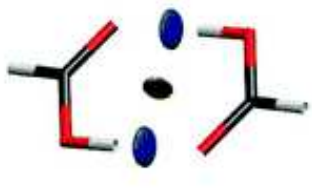
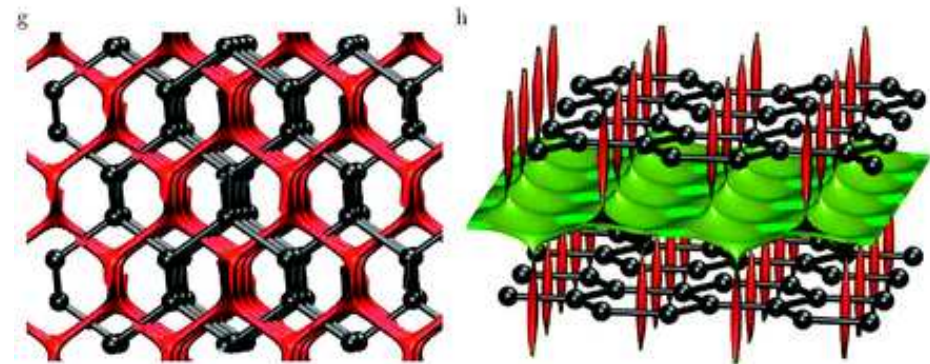

Fig. $4 \mathrm{NCI}$ isosurfaces $(s=0.5)$ for a) branched octane, b) bicyclo[2,2,2]octene, and the homomolecular dimers of c) benzene, d) methane, e) water, and f) formic acid. Gradient isosurfaces are also shown for cuboid sections of g) diamond and $h$ ) graphite. Color coding in the range -0.04 $<\operatorname{sign}\left(\lambda_{2}\right) \rho<+0.02$ au. Reprinted with permission from Ref. [50]. Copyright 2010 American Chemical Society.

Finally, in the benzene dimer (Figure 4c), there is an area of non-bonded overlap located at the center of each benzene ring, resembling the isosurface for bicyclo[2,2,2]octene. There is another lower-density surface between the overlapping portions of the benzenes, where $\pi$-stacking is expected. It is important to compare this image to the ones displayed in Table 1. The intermolecular interaction in benzene dimer appears very clearly with $\mathrm{NCI}$ as a surface that highlights the benzene to benzene stacking interaction, well beyond the pair interactions found with AIM. 


\section{Solids}

As we saw in the Introduction, non-covalent interactions in solid state have raised a great deal of interest lately, due to their relevance for self-assembly [36] and crystallization [37] processes.

Indeed, crystalline solids exhibit rich and challenging bonding patterns. We consider the prototypical examples of carbon in the diamond (Figure $4 \mathrm{~g}$ ) and graphite (Figure 4h) phases at their equilibrium geometries. In diamond, the carbon atoms are $s p^{3}$ hybridized and are connected by strong covalent bonds that form a tridimensional, tetrahedral network. Figure $4 \mathrm{~g}$ shows a NCI isosurface for a cuboid section of the diamond crystal. The non-covalent surface extends through the voids of the structure, creating a network similar to that of the covalent bonds.

Graphite in its $\alpha$ form (Figure $4 \mathrm{~h}$ ) has a bidimensional, hexagonal lattice, with the carbon atoms $s p^{2}$ hybridized and covalently bonded to their three nearest neighbors. The NCI isosurface shows areas of nonbonded overlap at the center of the hexagonal rings, as seen previously in benzene. $\pi-\pi$ stacking interactions between the graphene sheets are clearly manifested by the isosurfaces filling the interlayer spaces.

\section{Biological Systems}

Understanding of non-covalent interactions is crucial for the comprehension of the $3 \mathrm{D}$ structure and, thus, of the activity of biosystems. [65, 66] However, the calculation of the electron density in these systems is totally unbearable. Approximations need thus to be sought.

\subsection{Promolecular densities}

Densities are stable (see Section 3.2.2) to such an extent that NCI characteristics are already contained in the sum of atomic densities, $\rho_{i}^{a t}$. [63, 64] The resulting molecular density, also known as promolecular density, $\rho^{\text {pro }}$, is then given by:

$$
\rho^{\text {pro }}=\sum_{i} \rho_{i}^{a t}
$$

A promolecular density obtained from simple exponential atomic pieces is able to predict low-density, low-reduced-gradient regions similar to density-functional results. The free atomic densities used in these calculations consist of one Slatertype function for each electron shell, fit to closely reproduce spherically-averaged, density-functional atomic densities (see Appendix I). 
Approximate promolecular densities were constructed by summing exponential atomic densities for bicyclo[2,2,2]octene, and the homomolecular dimers of methane and water.

Resultant plots of $s^{\text {pro }}$ versus $\rho^{\text {pro }}$ for these species show the same 2D features seen in Figure 1. Also, 3D isosurfaces generated from the promolecular density (Figure 5) are very similar to those obtained previously with self-consistent DFT and even MP2 densities (Figure 4). For all cases considered, results at the self-consistent and promolecular level are qualitatively equivalent. Only slight quantitative differences are introduced by density relaxation that, as expected, shift the $s$ versus $\rho$ spikes to more bonding regimes. Specifically, a large shift toward smaller density values is observed in the spikes corresponding to non-bonded overlap, introducing less repulsion and greater stability. Taking this shift into account in the choice of isosurfaces, results at the self-consistent and promolecular level are qualitatively equivalent for all cases considered (see Figure 5 bottom). For example, lower cutoffs on the gradient (0.25-0.35) and higher cut-offs on the density (0.08-0.09 au) were required in order to generate the isosurface for bicyclo[2,2,2]octene.
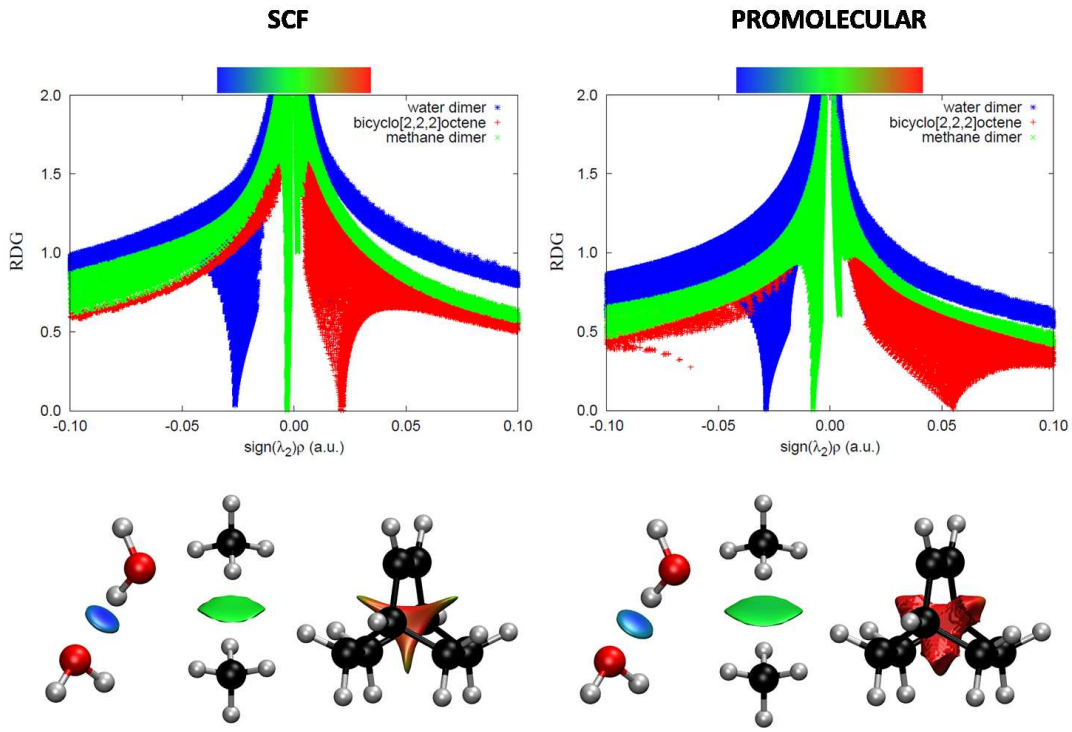

Fig. 5 Comparison between SCF and promolecular NCI results. The same $s(\rho)$ features are obtained using self-consistent (left) and promolecular (right) calculations, with a shift toward negative (stabilizing) regimes. Bottom: Taking the shift in spikes into account (i.e., changing the cut-off), the isosurface shapes remain qualitatively unaltered for selected small molecules. Figures are shown for both SCF (left) and promolecular densities (right). NCI surfaces correspond to $s=0.6$ and a color scale of $-0.03<\rho \operatorname{sign}\left(\lambda_{2}\right)<+0.03$ au for SCF densities. For promolecular densities, $s=$ 0.5 (water and methane dimers) or $s=0.35$ (bicyclo[2,2,2]octene), and the color scale is $-0.04<$ $\rho \operatorname{sign}\left(\lambda_{2}\right)<+0.04$ au. Reprinted with permission from Ref. Ref. [51]. Copyright 2011 American Chemical Society. 


\subsection{Examples}

Promolecular densities obviously lack relaxation; however, the promolecular densities are extremely useful in biomolecular systems, such as proteins or DNA. Because the calculation of the electron density in these systems becomes extremely computationally expensive, the promolecular density becomes an attractive option: noncovalent interactions can be analyzed with only the molecular geometry required as input.

We first consider two model polypeptides: an $\alpha$-helix consisting of 15 alanine residues and an anti-parallel $\beta$-sheet consisting of 17 glycine residues. Geometries of the polypeptides were obtained with the MMFF force field using the spartan program. [67] Both were capped with $\mathrm{COCH}_{3}$ and $\mathrm{NHCH}_{3}$ groups. Figure 6 displays low-gradient isosurfaces for cuboid regions at the center of these polypeptides, colored according to the corresponding density values. For the $\beta$-sheet, the lowestdensity portions of the gradient isosurface arise from hydrophobic, dispersiondominated interactions, primarily involving the $\mathrm{CH}_{2}$ groups of the glycines. The higher-density regions correspond to inter-residue hydrogen-bonds and repulsive interactions between the adjacent $\mathrm{C}=\mathrm{O}$ and $\mathrm{N}-\mathrm{H}$ groups. For the $\alpha$-helix, the isosurface has a large, low-density region within the helix and between the side-chain methyl groups. The higher-density portions of the isosurface correspond to interresidue hydrogen-bonds along the helix and repulsive interactions between adjacent $\mathrm{N}-\mathrm{H}$ groups.

We also considered the non-covalent interactions between nucleobases in the Bform of double-strand, six-base-pair (TGTGTG) DNA. The structure was obtained using the X3DNA program [68] with ideal geometry parameters. [69] Figure 6c displays the low-gradient isosurface for a cuboid section in the center of the DNA helix, colored according to the $\operatorname{sign}\left(\lambda_{2}\right) \rho$ values. The calculated isosurface resembles that of graphite, with broad, low-density regions indicative of $\pi$-stacking between base-steps. The interactions between individual deoxyadenosine-deoxythymidine and deoxycytidine-deoxyguanosine pairs are shown in Figure 6d,e. The isosurfaces show non-bonded overlap within the nucleobase rings, as in benzene and graphite, and hydrogen-bonding motifs similar to the formic acid dimer. The strong N-H $\cdots \mathrm{O}$ and $\mathrm{N}-\mathrm{H} \cdots \mathrm{N}$ hydrogen bonds can be clearly distinguished from the weaker, attractive $\mathrm{C}-\mathrm{H} \cdots \mathrm{O}$ interaction by the density values, as shown in different colors.

The hydrogen-bonding surfaces in the DNA model have density values of ca. $0.065 \mathrm{au}$, compared to density values of ca. $0.035 \mathrm{au}$ for the polypeptide hydrogen bonds. This is evident from the degree of blue shading for the hydrogen bonds in Figure 6. Since density values at hydrogen-bond critical points correlate with the interaction strength, $[25,70]$ our results indicate that the hydrogen bonds between nucleobase pairs are substantially stronger than between amino acids, in agreement with literature data. [71-73]

Let us now consider the interaction between a ligand and a protein active site. The low-gradient isosurface for a tetracycline inhibitor bound to the tetR protein in Figure 7 shows a complex web of non-covalent interactions between the ligand and active site. When analyzing non-covalent interactions in protein-ligand complexes, 

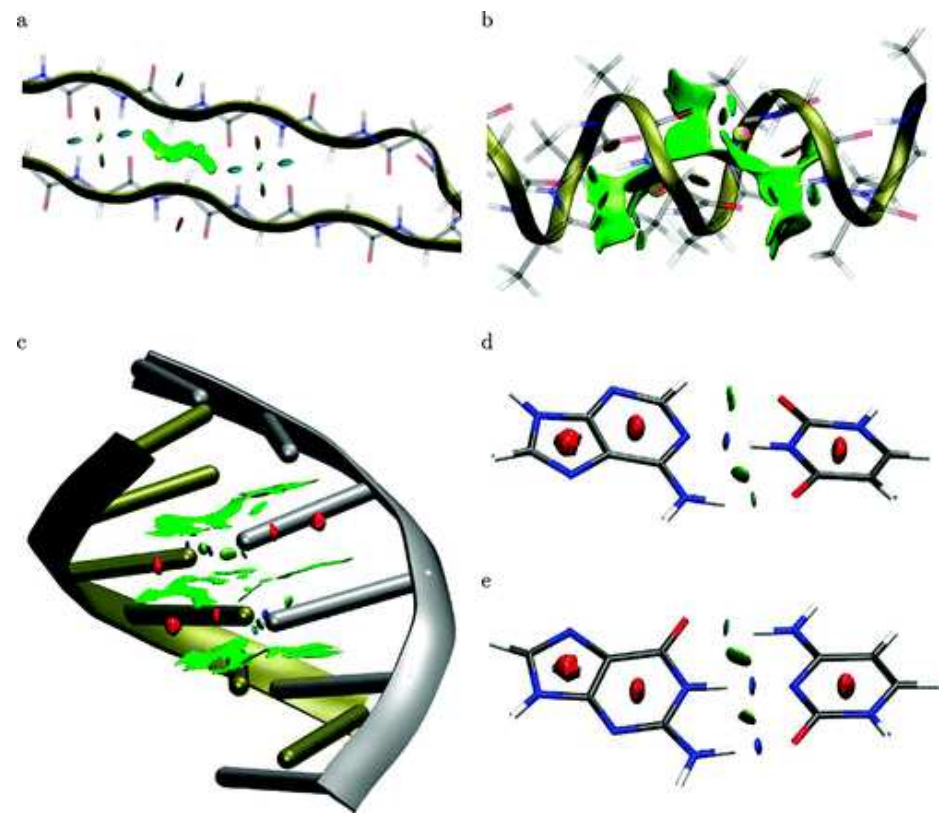

Fig. 6 Gradient isosurfaces $\left(s^{\text {pro }}=0.35\right.$ ) for cuboid sections of the (a) $\beta$-sheet and (b) $\alpha$-helix polypeptides. Gradient isosurfaces $\left(s^{\text {pro }}=0.25\right)$ are also shown for the (c) B-form of DNA, and the (d) A-T and (e) C-G base pairs. The surfaces are colored according to values of $\operatorname{sign}\left(\lambda_{2}\right) \rho$, ranging from -0.06 to +0.05 au. Reprinted with permission from Ref. [50]. Copyright 2010 American Chemical Society.

it is usually assumed that these interactions are due to a specific contact between two atoms. [44] However, it is clearly seen in Figure 7 that this assumption is only partly correct. Hydrogen bonds, such as those between the tetracycline amine groups and two water molecules (shown in orange), are directional and specific. Conversely, van der Waals, dipole-dipole, and hydrophobic interactions, such as those between the tetracycline and the Leu61, Val91, Ile136, and Val166 residues (shown in yellow), are not atom-specific and occupy broader regions in space. The figure reveals some steric clashes (orange and red regions of the isosurface) that must be offset by stronger, attractive interactions to give binding in this crystal structure. A ligand "fits" the geometry of the active site, and the interaction energy between the ligand and protein is comprised of many small contributions. When trying to design a new ligand to fit a specific active site, one should consider all such interactions. 


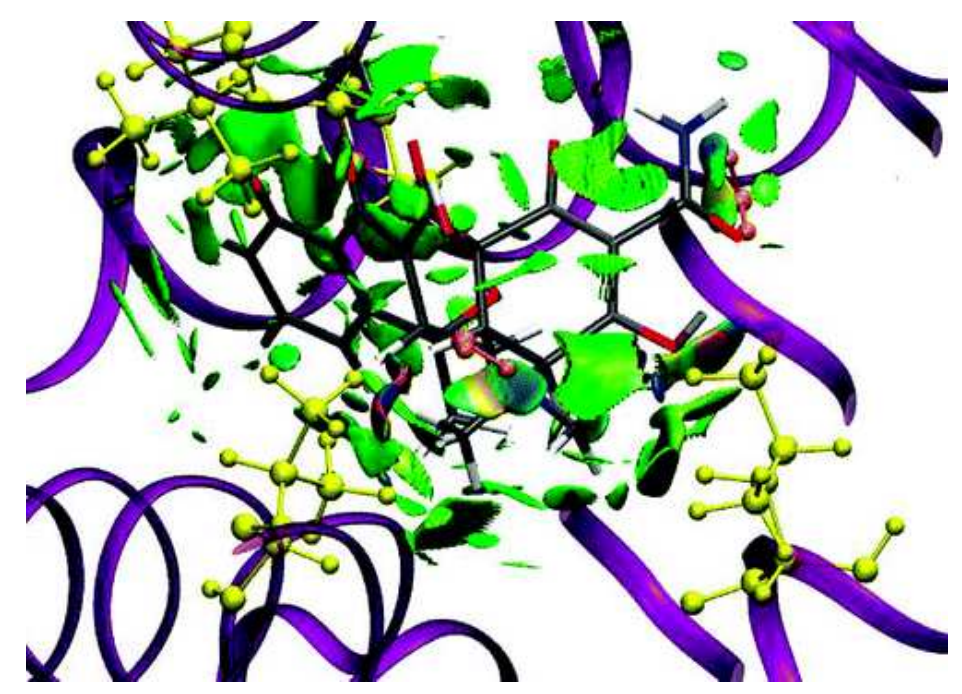

Fig. 7 Gradient isosurfaces $\left(s_{\text {pro }}=0.35\right.$ ) for interaction between the tetR protein and tetracycline inhibitor. Surfaces colored in the $\operatorname{sign}\left(\lambda_{2}\right) \rho$ range from -0.06 to +0.05 au. Reprinted with permission from Ref. [50]. Copyright 2010 American Chemical Society. 


\section{New bonds: do we really need to name them all?}

Hydrogen bonding was postulated in the early $20^{\text {th }}$ century based on the stunning macroscopic differences between the first and second row hydrides, i.e. water is a high boiling liquid without, which there would be no life and hydrogen sulphide is a stinking gas, under ambient conditions. [1]

With the advent of molecular beam and cryogenic experimental methods as well as the ever advancing theoretical methods, HBs have been proved to exist in $\mathrm{H}_{2} \mathrm{~S}$ as well. [74] But things go even further. About a century later, chemists have identified a wealth of new bonding types along the periodic table. Halogen bonds (XBs) (group 17) are frequently exploited for crystal engineering. [75] Recently, similar bonding mechanisms have been proposed for adjacent main-group elements, and noncovalent "chalcogen bonds" (group 16) [76] and "pnictogen bonds" (group 15) [77] have also been identified in crystal structures. Recently, even carbon bonding (group 14) [78] has been proposed as a stabilizing interaction.

One of the most interesting features of NCI is that it is based on the density. Thus, it is expected to be able to reveal any type of bonding. Before and beyond its corresponding identification and characterization, the signature on the electron density will be present. In the coming sections we briefly show some representatives of the new bonds series, highlighting the possibility to reveal interactions all along the periodic table from just the fast analysis of NCI.

\subsection{Halogen bonding}

Halogen bonds (XBs) occur between a halogen atom, playing the role of Lewis acid, and a Lewis base. This non-covalent interaction is analog to hydrogen bonding in the sense that in both cases, an atom or group of atoms with high electron density donates charge to an acceptor which is electron poor. Similar to HBs, XBs are also anisotropic and involved in various fields, such as supramolecular chemistry or even materials chemistry. Moreover, halogenated coumpounds are often encountered in medicinal chemistry [79] and drug discovery. [80] Quantum Chemistry approaches have revealed a sigma-hole along the axis defined by the halogen atom and the acceptor. This can be studied thanks to the Molecular Electrostatic Potentials, showing the charge distribution as well as the nature of the interaction as electrostatics and charge transfer driven.

We have analyzed $\mathrm{NCI}$ in a series of trifluoromethyl halides, $\mathrm{CF}_{3} \mathrm{X}$, where $\mathrm{X}=$ $\mathrm{Cl}, \mathrm{Br}$,I with dimethyl ether (DME), dimethyl sulfide (DMS), trimethyl phosphine (TMP) and imidazolin-2-ylidene (NHC). All systems were optimized using secondorder Møller-Plesset perturbation theory with the aug-cc-pVDZ(-PP) basis set. [81] The wavefunctions were obtained at DFT level using the B3LYP functional and the $6-31++G^{* *}$ basis set except for iodine, where the pseudopotential LANL2DZ was used. 
In the first four cases, the 2D plots (Figure 8) clearly show a spike at vert low density, corresponding to a typical van der Waals interaction. In Figure 8b, a second spike arises, exhibiting non bonding interactions between the chlorine atom and DMS hydrogens. They are closer to the halogen than the sulfide atom and benefit from the high electron density at the halogen.

In agreement with previous studies, the prominent spike is shifted to larger electron densities (left) for iodine, reflecting a stronger non bonding interaction (see Figures 8e-f) as $\mathrm{X}$ molecular weight increases.

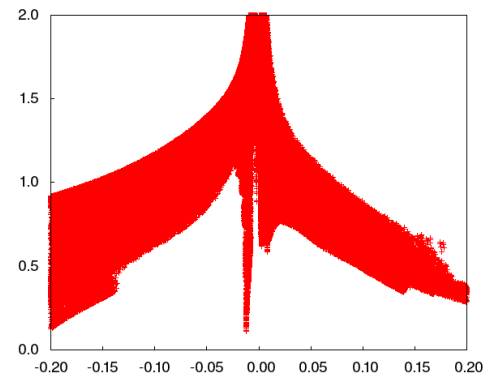

a) $\mathrm{CF}_{3} \mathrm{Cl}-\mathrm{DME}$

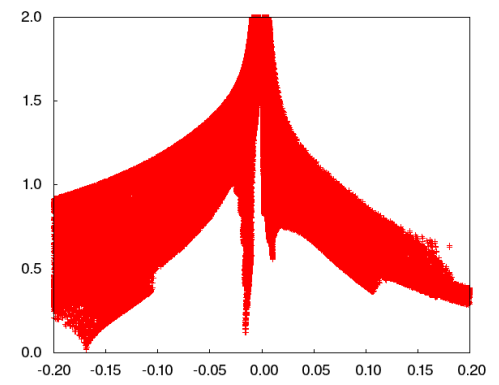

c) $\mathrm{CF}_{3} \mathrm{Br}-\mathrm{DME}$

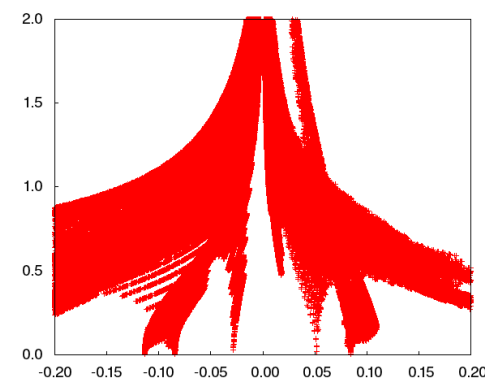

e) $\mathrm{CF}_{3} \mathrm{I}-\mathrm{NHC}$

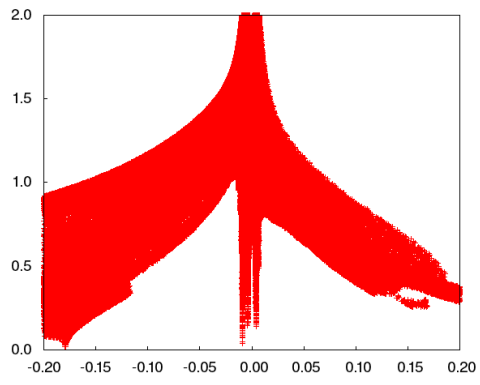

b) $\mathrm{CF}_{3} \mathrm{Cl}-\mathrm{DMS}$

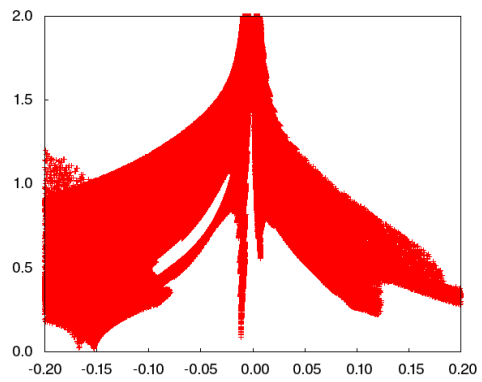

d) $\mathrm{CF}_{3} \mathrm{Br}-\mathrm{TMP}$

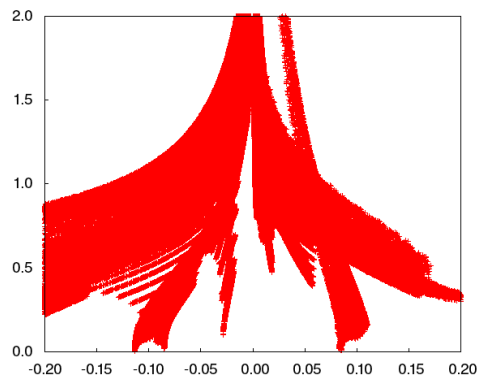

f) $\mathrm{CF}_{3}$ I-TMA

Fig. 8 2D plots for halogen bonded complexes: a) $\mathrm{CF}_{3} \mathrm{Cl}-\mathrm{DME}$ b) $\mathrm{CF}_{3} \mathrm{Cl}-\mathrm{DMS}$ c) $\mathrm{CF}_{3} \mathrm{Br}-\mathrm{DME}$ d) $\mathrm{CF}_{3} \mathrm{Br}-\mathrm{TMP}$ e) $\mathrm{CF}_{3} \mathrm{I}-\mathrm{NHC}$ f) $\mathrm{CF}_{3} \mathrm{I}-\mathrm{TMA}$. See Section 7.5.2 for an interpretation of iodine compounds extra peaks. 


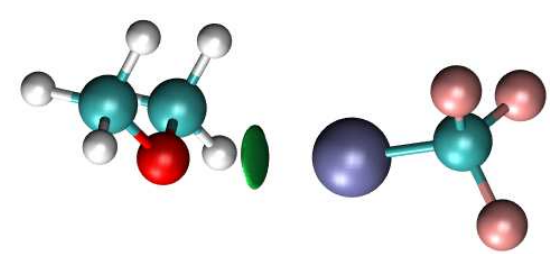

a) $\mathrm{F}_{3} \mathrm{Cl}-\mathrm{DME}$

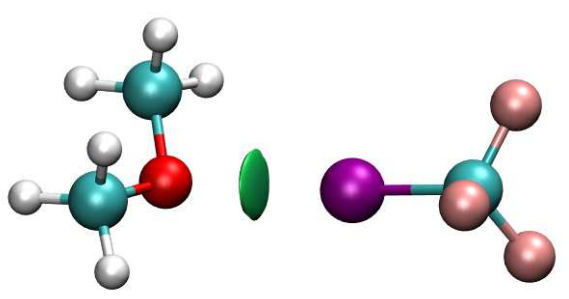

c) $\mathrm{CF}_{3} \mathrm{Br}-\mathrm{DME}$

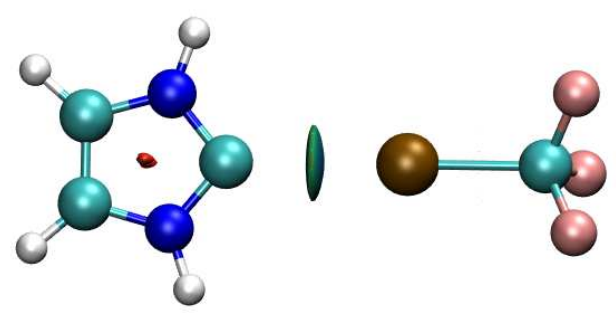

e) $\mathrm{CF}_{3} \mathrm{I}-\mathrm{NHC}$

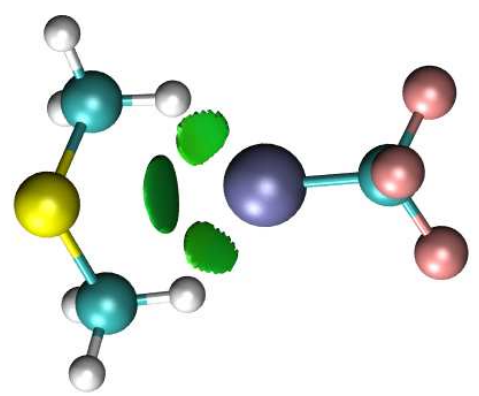

b) $\mathrm{CF}_{3} \mathrm{Cl}-\mathrm{DMS}$

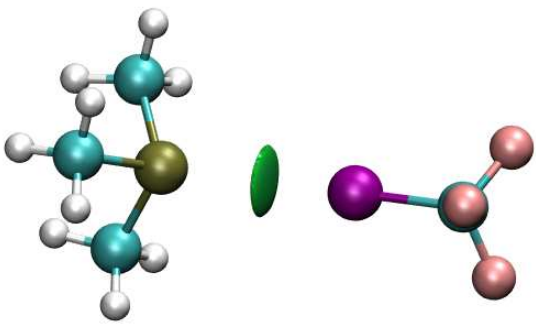

d) $\mathrm{CF}_{3} \mathrm{Br}-\mathrm{TMP}$

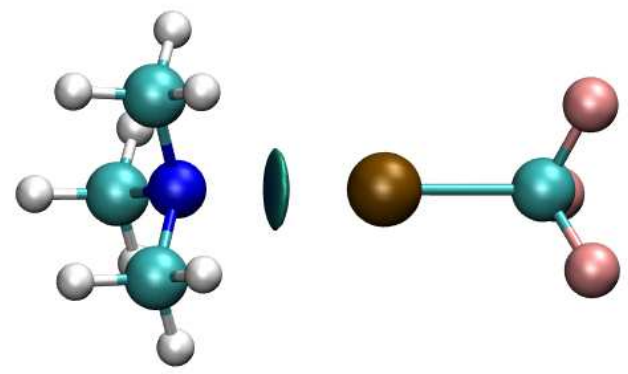

f) $\mathrm{CF}_{3} \mathrm{I}-\mathrm{TMA}$

Fig. 9 3D plots for halogen bonded complexes: a) $\mathrm{CF}_{3} \mathrm{Cl}$-DME b) $\mathrm{CF}_{3} \mathrm{Cl}-\mathrm{DMS}$ c) $\mathrm{CF}_{3} \mathrm{Br}-\mathrm{DME}$ d) $\mathrm{CF}_{3} \mathrm{Br}-\mathrm{TMP}$ e) $\mathrm{CF}_{3} \mathrm{I}-\mathrm{NHC}$ f) $\mathrm{CF}_{3} \mathrm{I}-\mathrm{TMA}$. NCI isosurfaces correspond to $s=0.5$ au and a colorscale of $-0.04<\operatorname{sign}\left(\lambda_{2}\right) \rho<+0.04$ au.

\subsection{Pnictogen bonding}

Pnictogen bonding is a weak non-covalent bonding involving group 15 elements as electron density acceptors. Similiar to halogens, the pnictogen atoms possess a sigma-hole: a region of positive electrostatic potential in the direction of the bond, 


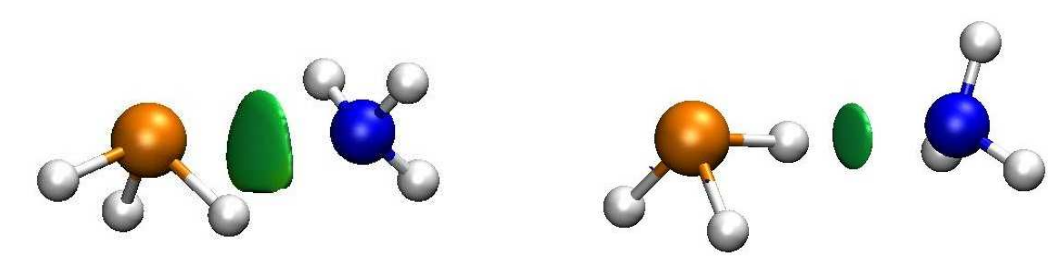

$\mathrm{NH}_{3}-\mathrm{PH}_{3}$
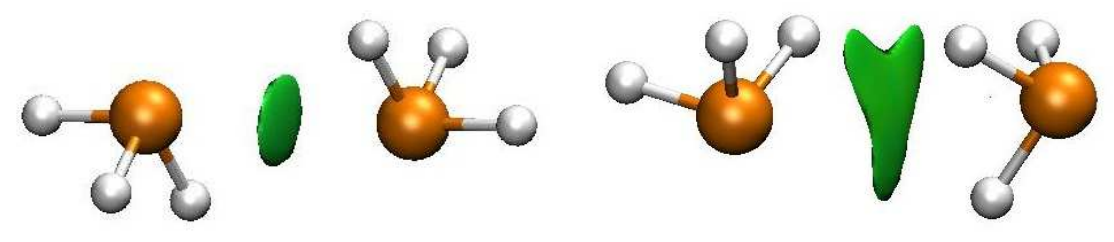

$\mathrm{PH}_{3}-\mathrm{PH}_{3}$

Fig. 10 Pnictogen bond examples: a-b) $\mathrm{NH}_{3}-\mathrm{PH}_{3}$, c-d) $\mathrm{PH}_{3}-\mathrm{PH}_{3}$. 3D isosurface was produced using the cut-off values of $s=0.5$ and $\rho<0.05$.

which is attracted to a lone pair on a nucleophile with an outer negative electrostatic potential. [82,83]

Pnictogen bonding is present in the complex between $\mathrm{NH}_{3}$ and $\mathrm{PH}_{3}$ (Figure 10a) [77] where the $\mathrm{N}$ atom is the donor of electron density. The sigma-hole gives rise to the equilibrium geometries: the two molecules are oriented such that the $\mathrm{P}$ and $\mathrm{N}$ atoms face one another directly, without the intermediacy of a $\mathrm{H}$ atom. This attraction is due in part to the transfer of electron density from the lone pair of the $\mathrm{N}$ atom to the $\sigma^{*}$ antibond of a P-H covalent bond. Unlike in hydrogen bonds, the pertinent hydrogen is oriented about $180^{\circ}$ away from the $\mathrm{N}$ (instead of toward), and the $\mathrm{N}$ lone pair overlaps with the lobe of the $\mathrm{P}-\mathrm{H} \sigma^{*}$ orbital that is closest to the $\mathrm{P}$. The calculated binding energy of this pnictogen-bonded complex is more stable than the hydrogen-bonded complex that is formed between the same two molecules where the $\mathrm{N}$ atom is the proton acceptor (Figure 10b).

We have optimized the $\mathrm{NH}_{3}-\mathrm{PH}_{3}$ and $\mathrm{PH}_{3}-\mathrm{PH}_{3}$ pairs at the $\omega \mathrm{B} 97 \mathrm{X}-\mathrm{D} / 6-31+\mathrm{G}^{*}$ level of theory. The $\mathrm{NCI}$ analysis of the $\mathrm{NH}_{3}-\mathrm{PH}_{3}$ complexes shows the presence of non-covalent bonding and this is illustrated by the $3 \mathrm{D}$ isosurfaces of both complexes (Figure 10a-b). The green color of both interactions indicates that the two types of bonding have similar bonding strengths corresponding to that of van der Waals interactions. Whereas in the H-mediated complex, a typical picture of HB is obtained (Figure 10b), a thick surface is obtained in the case of the pnictogen bonding (Figure 10a), which is extended like in the case of van der Waals, but thick like HB ones.

It is interesting to note that in contrast to halogen bonds, there is no requirement of a sigma-hole of positive electrostatic potential on the $\mathrm{P}$ atom, nor is it necessary for the two interacting atoms to be of differing potential. In fact, the two atoms can be identical, as the global minimum of the $\mathrm{PH}_{3}$ homodimer has the same structure, 
characterized by a $\mathrm{P} \cdots \mathrm{P}$ attraction. Indeed, for the complex between $\mathrm{PH}_{3}$ and $\mathrm{PH}_{3}$, the $\mathrm{P}$ atoms possess a partial positive charge and none of the located minima found on the potential energy surface correspond to a hydrogen-bonded complex. [77] The two minima that were located correspond to complexes where the $\mathrm{P}$ atoms approach one another (Figure 10c-d). The complex with the symmetric geometry (Figure 10c) was found to be dominated by electrostatic interactions, corresponding to pnictogen bonding, whereas the second structure (Figure 10d) was found to be dominated by dispersion. This shows in the NCI isosurfaces where the interaction region in Figure $10 \mathrm{~d}$ occupies a larger volume than that of Figure 10c. This is in agreement with the more diffuse character of the dispersion interaction compared to the pnictogen bond which is more concentrated along the bonding direction.

\subsection{Carbon bonding}

Recently, inspired by the identification of halogen, chalcogen and pnictogen bonding, Mani et al. [78] investigated whether carbon, being a positive centre, can accept electron density. Indeed, both experimental and theoretical studies agree that the tetrahedral face of methane can act as a hydrogen bond acceptor. Rotational spectra of complexes like $\mathrm{CH}_{4} \cdots \mathrm{HF}, \mathrm{CH}_{4} \cdots \mathrm{HCl}, \mathrm{CH}_{4} \cdots \mathrm{HCN}$ and $\mathrm{CH}_{4} \cdots \mathrm{H}_{2} \mathrm{O}$ further confirm this fact. [84] While the tetrahedral face of methane has an electron rich centre and can act as a hydrogen bond acceptor, substitution of one of its hydrogens with some electron withdrawing group (such as $-\mathrm{F} / \mathrm{OH}$ ) can make the opposite face electron deficient. The complex between $\mathrm{CH}_{3} \mathrm{~F}$ and $\mathrm{H}_{2} \mathrm{O}$ has a potential energy minimum with water oxygen pointing towards the tetrahedral face of $\mathrm{CH}_{3}$. Similar interactions are also found for several methanol complexes in which the electron deficient atom (oxygen) interacts with one of the water's lone pair.

Four examples of complexes, which represent minima on the potential energy surface, are shown in Figures 11a-d. All molecules and bimolecular complexes were optimized at the $\omega \mathrm{B} 97 \mathrm{X}-\mathrm{D} / 6-31+\mathrm{G}^{*}$ level of theory. In these complexes, the electron density donors ( $\mathrm{O}, \mathrm{P}, \mathrm{S}$ and $\mathrm{F}$ atoms) are oriented towards the $\mathrm{CH}_{3}$ face of methanol. Through NCI analysis, the presence of intermolecular interaction is evident in all the complexes. The weak nature of this type of interaction is indicated by the green color of the NCI-isosurfaces that corresponds to van der Waals interactions.

It should be noted that, in spite of the weakness of these type of interactions, they are extremely relevant, since these and similar interactions could give an enthalpic contribution to the so called "hydrophobic interactions". [78] 


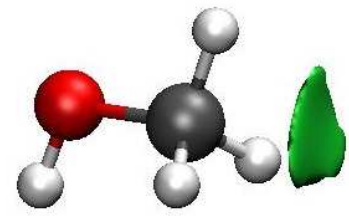

a) $\mathrm{MeOH}-\mathrm{H}_{2} \mathrm{O}$

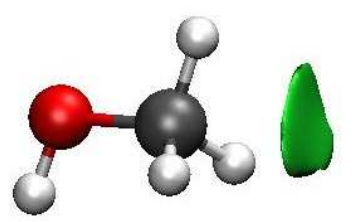

c) $\mathrm{MeOH}-\mathrm{SH}_{2}$
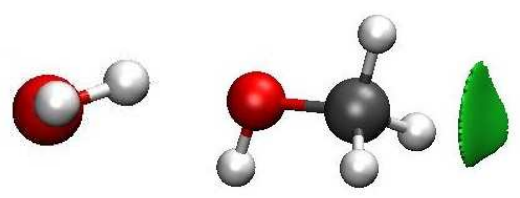

b) $\mathrm{MeOH}-\mathrm{PH}_{3}$
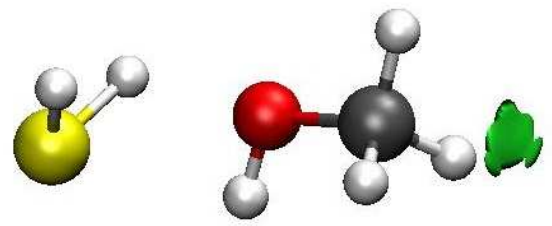

d) $\mathrm{MeOH}-\mathrm{FH}$

Fig. 11 Carbon bond examples for several electron donors (O, P, S and F): a) $\mathrm{MeOH}-\mathrm{H}_{2} \mathrm{O}$ b) $\mathrm{MeOH}-\mathrm{PH}_{3}$ c) $\mathrm{MeOH}-\mathrm{SH}_{2}$ d) MeOH-FH. 3D isosurface was produced using the cut-off values of $s=0.5$ and $\rho<0.05$.

\subsection{The di-hydrogen bond}

Very recently the new term di-hydrogen bond was coined to describe an interaction of the type D-H $\cdots$ H-E, where D is a typical hydrogen donor such as $\mathrm{N}$ or O. The interesting thing about this type of bond, is that the acceptor atom is also a hydrogen.

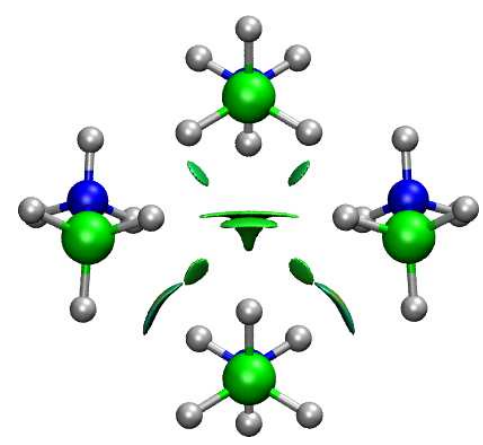

a) Gaseous phase

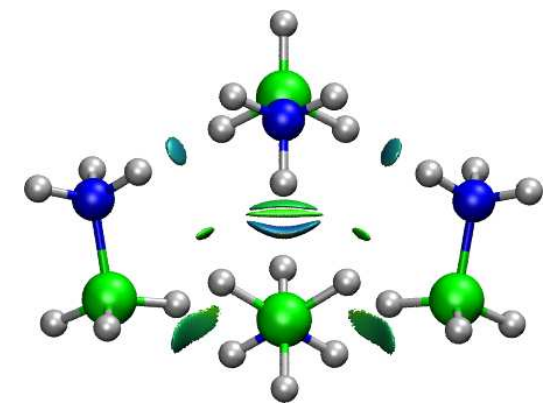

b) Crystal structure

Fig. 12 Dihydrogen interactions in a $\mathrm{BH}_{3} \mathrm{NH}_{3}$ tetramer in a) the fully optimized gas-phase geometry and b) the solid-state geometry. NCI surfaces correspond to $s=0.4$ au and a color scale of -0.03 $<\operatorname{sign}\left(\lambda_{2}\right) \rho<+0.03$ au. Reprinted with permission from Ref. [51]. Copyright 2011 American Chemical Society. 
Thus, the accepting hydrogen atom must be negatively charged and $\mathrm{E}$ has to be an atom capable of accommodating a hydridic hydrogen. Transition metals and boron are some known examples of atoms occurring at position E. Within di-hydrogen bonded complexes, $\mathrm{BH}_{3} \mathrm{NH}_{3}$ is perhaps the most widely studied. [86-88] We have analyzed the tetramer $\left(\mathrm{BH}_{3} \mathrm{NH}_{3}\right)_{4}$, whose geometry has been derived from the solid state. Figure 12 shows the NCI results for $\left(\mathrm{BH}_{3} \mathrm{NH}_{3}\right)_{4}$. It can be seen that each $\mathrm{BH}_{3} \mathrm{NH}_{3}$ molecule interacts with the surrounding ones establishing one di-hydrogen bond with each, and numerous van der Waals contacts. The surfaces obtained are, in all cases, completely analogous to those obtained in previous examples for hydrogen bonds.

We have also studied a series of nine complexes presenting di-hydrogen bonds ( $\mathrm{LiH}-\mathrm{HCCH}, \mathrm{LiH}-\mathrm{HCN}, \mathrm{LiH}-\mathrm{HCF}_{3}, \mathrm{NaH}-\mathrm{HCCH}, \mathrm{NaH}-\mathrm{HCN}, \mathrm{NaH}-\mathrm{HCF}_{3}, \mathrm{HBeH}-$ $\mathrm{HCCH}, \mathrm{HBeH}-\mathrm{HCN}, \mathrm{HBeH}-\mathrm{HCF}_{3}$ ) to check the ability of NCI to detect new types of bonds even at the promolecular level. After using the MAPS Platform [89] to set up the initial systems, the complexes were optimized at the MP2/aug-cc-pVDZ level with NWChem. [90] The optimized coordinates were used to perform NCI promolecular analysis. The results are displayed in Figure 13.

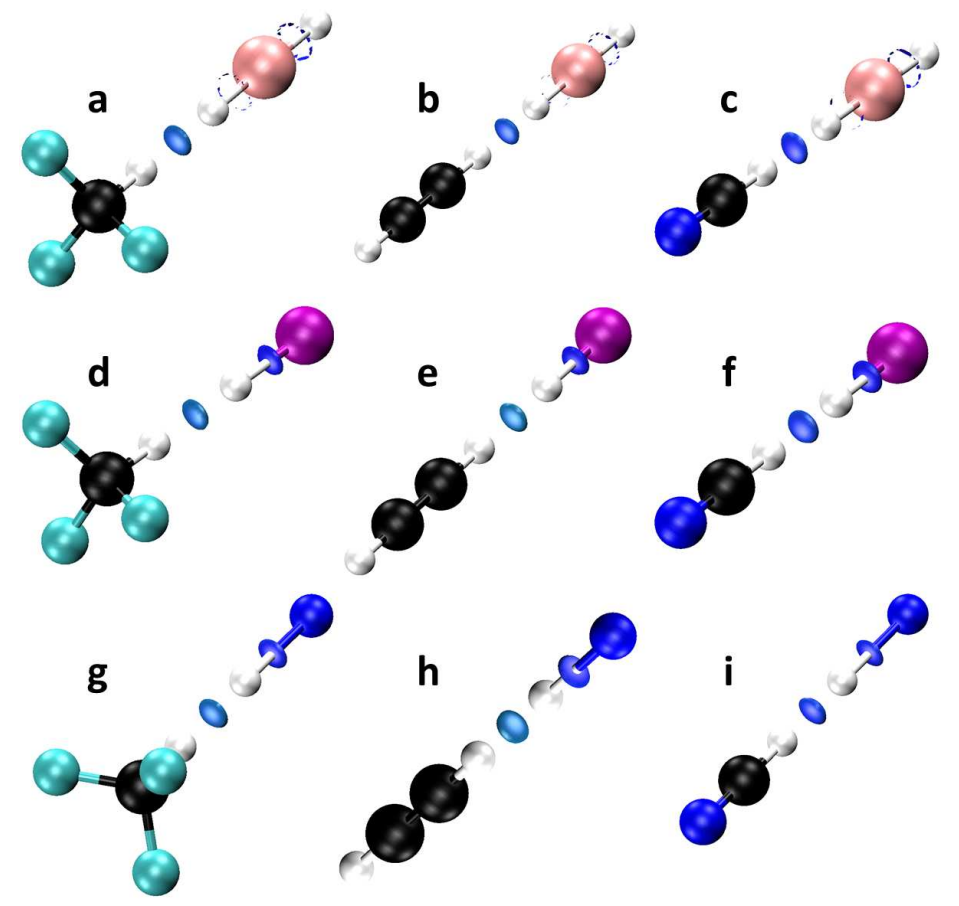

Fig. 13 NCI surfaces for several di-hydrogen complexes: a) $\mathrm{HBeH}-\mathrm{HCF}_{3}$ b) $\mathrm{HBeH}-\mathrm{HCCH}$ c) $\mathrm{HBeH}-\mathrm{HCN}$ d) $\mathrm{LiH}-\mathrm{HCF}_{3}$ e) $\mathrm{LiH}-\mathrm{HCCH}$ f) $\mathrm{LiH}-\mathrm{HCN}$ g) $\mathrm{NaH}_{-} \mathrm{HCF}_{3}$ h) $\mathrm{NaH}-\mathrm{HCCH}$ i) $\mathrm{NaH}-$ HCN. The NCI isosurfaces were plotted for $s=0.3$ and a color scale of $-0.03<\operatorname{sign}\left(\lambda_{2}\right) \rho<$ +0.03 . 
Even at this rough level, NCI allows to follow the evolution of the interaction strength for the different systems. The interaction basins appear similar to those from strong hydrogen bonds. They also seem to be stronger than the di-hydrogen bond in the $\mathrm{BH}_{3} \mathrm{NH}_{3}$ complex. It should be noted that this agrees with the fact that di-hydrogen bonds have been attributed very variable strengths. Crabtree et al. [91] have placed the $\mathrm{NH} \cdots \mathrm{HB}$ contact at the upper end of the energy range quoted for hydrogen bonds. Popelier instead, has found it to be in the range of normal HB strengths [86] and Morrison and Siddick [87] assigned it towards the lower end of the hydrogen bond strength spectrum. Our results show that the range of energies covered go (at least) from the strong to the medium HB-type of strength.

\subsection{Metal driven interactions}

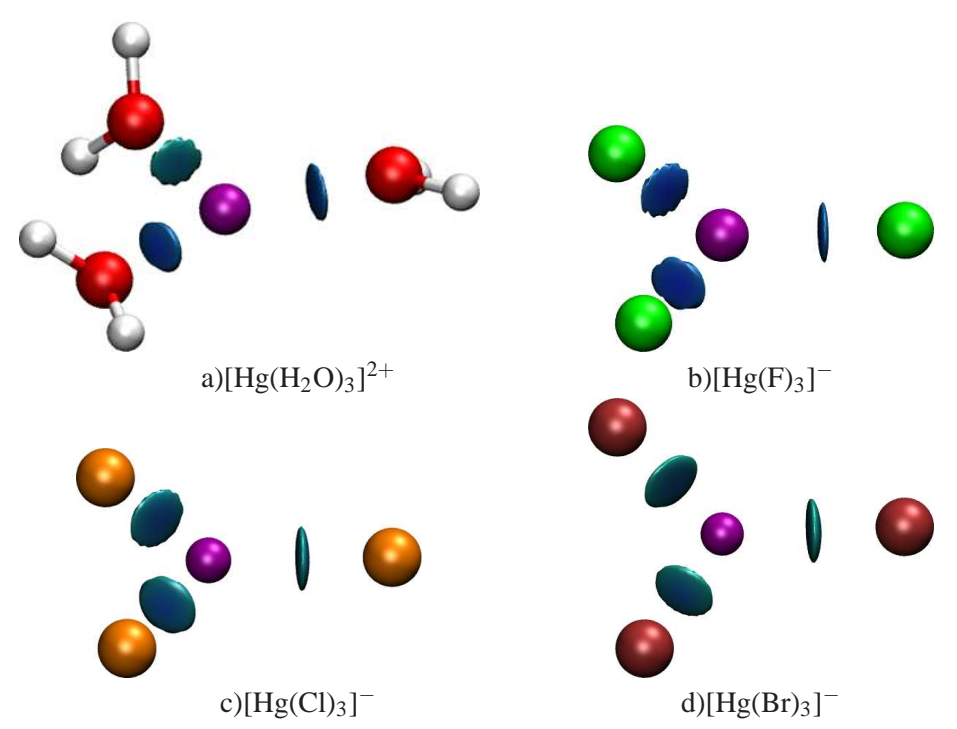

Fig. 14 Complexation of $\mathrm{Hg}$. a) $\left[\mathrm{Hg}\left(\mathrm{H}_{2} \mathrm{O}\right)_{3}\right]^{2+}$ b) $\left[\mathrm{Hg}(\mathrm{F})_{3}\right]^{-} \quad$ c) $\left[\mathrm{Hg}(\mathrm{Cl})_{3}\right]^{-}$d) $\left[\mathrm{Hg}(\mathrm{Br})_{3}\right]^{-}$. Reprinted with permission from Ref. [51]. Copyright 2011 American Chemical Society.

Although NCI is usually used for weak interactions, its basis does not limit the tool to only weak interactions. Indeed, it can be used for covalent and ionic interactions, as well (see S.I. in Ref. [50] and Ref. [61]). Most commonly, it is also used within metallorganic frameworks to detect interactions with metals. We will analyze several model examples relelvant to solvation and protein structure. 


\subsection{1 $\mathrm{Hg}^{2+}$ complexation}

Understanding the complexation of ions and their preferential ligands is of prime relevance when studying their bioactivity. As an example, their absorbance and transport through the body is extremely dependent on their complexation. It would then be extremely interesting to be able to identify the main series as well as the potential substitution sites. In addition Mercury(II) is a heavy metal cation which is specially challenging for quantum mechanical treatment as both correlation and relativistic effects play a crucial role in modeling its complexes.

Figures 14a-d illustrate the ability of NCI to visualize in a fast and efficient manner the complexation sites of $\mathrm{Hg}$ and to discriminate the strength of the binding energies between the cation and its ligands. Figure 14a shows the $\left[\mathrm{Hg}\left(\mathrm{H}_{2} \mathrm{O}\right)_{3}\right]^{2+}$ complex and suggests that the three waters bound to $\mathrm{Hg}$ are not equivalent, one of the water molecules being more weakly bonded than the others. In order to corroborate this observation, it is possible to perform a decomposition of the interaction energy of such a complex using the RVS [85] (Reduced Variational Space) procedure. Both polarization and charge transfer are significantly weaker for one water molecule: whereas two of the water molecules show a polarization energy of ca. $-15 \mathrm{kcal} / \mathrm{mol}$, and a charge transfer energy of $-10 \mathrm{kcal} / \mathrm{mol}$, the third water shows a stabilization due to polarization and charge transfer by only $-12.7 \mathrm{kcal} / \mathrm{mol}$ and -6.2 $\mathrm{kcal} / \mathrm{mol}$, respectively.

The ability of NCI to recover the ordering of ligands is also applicable when different ligand series are analyzed. Figure $14 \mathrm{~b}-\mathrm{d}$ show $\left[\mathrm{Hg}(\mathrm{X})_{3}\right]$ complexes $(\mathrm{X}$ standing for $\mathrm{F}, \mathrm{Cl}$ and $\mathrm{Br}$ ). It is clearly seen that $\mathrm{F}$ is more strongly bonded than $\mathrm{Cl}$ and $\mathrm{Br}$. This result is in agreement with their binding energies, which are -632.8 , -571.0 and $-562.6 \mathrm{kcal} / \mathrm{mol}$, respectively.

\subsection{2 $\mathrm{Zn}^{2+}$ fingers}

Metals play a decisive role in many protein active sites as cofactors. The zinc finger is a small protein structural motif that can be found in many biological systems. It is characterised by the fact than one or more zinc ions can stabilize the fold of a protein. One or two $\mathrm{Zn}$ (II) cations [100] are often tetrahedrally coordinated to four or six amino acids such as cysteine (Cys) or histidine (Hys) forming four major cores: $\mathrm{ZnCys}_{4}, \mathrm{ZnCys}_{3} \mathrm{His}_{2} \mathrm{ZnCys}_{2} \mathrm{His}_{2}$ and $\mathrm{ZnCys}_{6}$. [101]

Starting from the $\mathrm{ZnCys}_{4}$ core, its modeling can be carried out by substitution of Cys by methyl thiolate, $\mathrm{CH}_{3} \mathrm{~S}^{-}$. [101] In order to analyse the effect of the environement within the HSAB theory, we have analyzed the series $\mathrm{M}^{2+}\left[\mathrm{SCH}_{3}\right]_{4}^{2-}$, with $\mathrm{M}=\mathrm{Mg}, \mathrm{Zn}, \mathrm{Pd}$ (from hard to soft Lewis acids). All complexes were optimized at the $\omega \mathrm{B} 3 \mathrm{LYP} / 6-31++\mathrm{G}^{* *}$ level of theory except for Pd, which was optimized with the pseudopotential LANL2DZ.

In all cases, the tetracoordination of the metal to the XMe ligands clearly stands out as a strong interaction (deep blue in Figure 15). It is interesting to note that the interaction spike shifts from ca. $\operatorname{sign}\left(\lambda_{2}\right) \rho=-0.03$ au. to $\operatorname{sign}\left(\lambda_{2}\right) \rho=-0.06$ au. when 


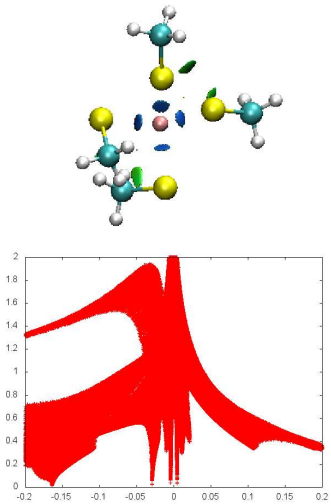

a) $\left[\mathrm{Mg}(\mathrm{SMe})_{4}\right]^{2-}$
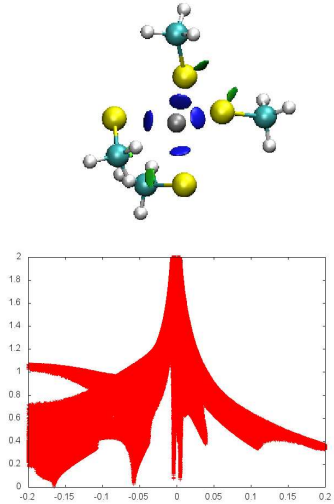

b) $\left[\mathrm{Zn}(\mathrm{SMe})_{4}\right]^{2-}$
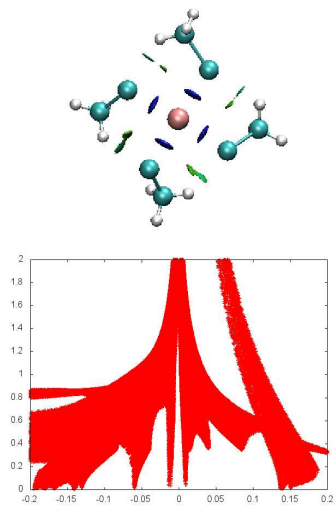

c) $\left[\mathrm{Pd}(\mathrm{SMe})_{4}\right]^{2-}$

Fig. $15 \mathrm{NCI}$ plots for $\mathrm{Zn}$ finger model: a) $\left[\mathrm{Mg}(\mathrm{SMe})_{4}\right]^{2-}$, b) $\left[\mathrm{Zn}(\mathrm{SMe})_{4}\right]^{2-}$, c) $\left[\mathrm{Pd}(\mathrm{SMe})_{4}\right]^{2-}$. Top: 3D NCI plot. Bottom: 2D NCI plot.

passing from $\mathrm{Mg}(\mathrm{II})$, which is a hard cation, to $\mathrm{Zn}(\mathrm{II})$ and $\mathrm{Pd}(\mathrm{II})$, which are intermediate and soft cations. This is in agreement to what is expected from the nature of the $\mathrm{S}$ bridge (soft) to the ligands within the HSAB theory.

Spikes appear at very low densities which correspond to secondary interactions in between the ligands, which stabilize the whole structure. As a final note, the big spikes appearing for $\mathrm{Pd}$ at ca. $\rho=0.1$, along with the peaks appearing for the iodine compounds in Section 7.1, are an artifact from the pseudopotential, and as such, should be disregarded. 


\section{Reactivity}

Understanding and predicting chemical reactivity are some of the achievements of quantum chemistry. In this regard, the Woodward-Hoffmann rules [21] for pericyclic reactions have become a classical reference. By definition, pericyclic reactions evolve via a cyclic aromatic transition state of delocalized electrons where bond making and bond breaking occur simultaneously in a cyclic array. Using the orbital symmetry conservation, Woodward-Hoffmann proposed a list of rules of thumb able to predict the mechanism and, hence the stereoselectivity of pericyclic reactions. Examples include cycloadditions, electrocyclizations, sigmatropic rearrangements, and chelotropic reactions. Much work has been devoted to show that electron circulation on the pericyclic transition states may be smartly characterized by the topology of the electron localization function (ELF). [92] Recently, it was shown how the combined analysis of the NCI method and ELF may be employed as a visual tool to understand the electron reorganization along an intrinsic reaction path (IRC). [93] Contrary to ELF, the reduced density gradient does no suffer from catastrophes (sudden creation and/or destruction of critical points), being possible to preclude the bonding formation from the first stages of the reaction.

One example of application of NCI to predict the outcome of pericyclic reactions is provided by the thermal ring-opening of cis- and trans- 1,2,3,4-tetrafluoro1,4-bis(pentafluorsulfanyl)cyclobutene (see Figure 16). As a thermal, $4 n$ electron process, the Woodward-Hoffmann rules predict that the conrotatory opening is more favorable than the disrotatory one. [21,94] Additionally, a given terminal substituent may either rotate "outward", leading to $(E, E)$-1,2,3,4-tetrafluoro-1,4bis(pentafluorosulfanyl)butadiene or "inward" to yield $(Z, Z)-1,2,3,4$-tetrafluoro-1,4bis(pentafluorosulfanyl)butadiene. Activation energies obtained at the $\omega \mathrm{B} 97 \mathrm{X}-\mathrm{D} / 6-$ $31 \mathrm{G}^{* *}$ level for $(E, E)$ and $(Z, Z)$ transition states are $41.55 \mathrm{kcal} / \mathrm{mol}$ and 21.12 $\mathrm{kcal} / \mathrm{mol}$, respectively. Because this kind of stereoselectivity is related to the direction of the twist, it was named torquoselectivity by Houk and co-workers. [95]

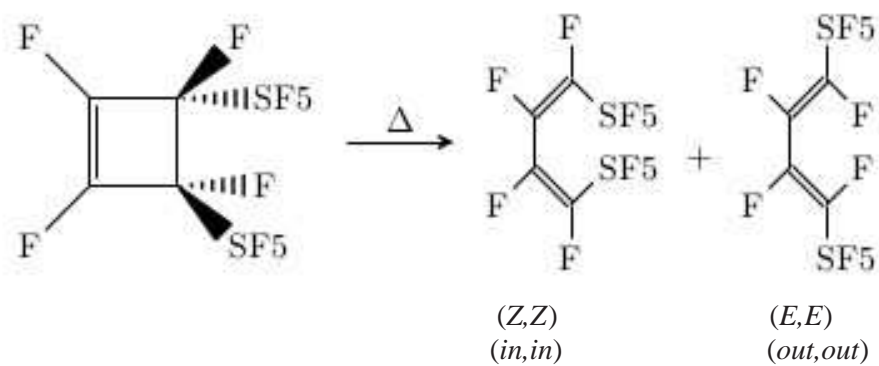

Fig. 16 "Outward" (out) and "inward" (in) conrotatory processes for the thermal ring opening of trans-1,2,3,4-tetrafluoro-1,4- bis(pentafluorsulfanyl)cyclobutene. 
Rondan and Houk proposed in 1984 a widely accepted orbital model able to explain torquoselectivity. $[96,97]$ In a nutshell, this model states that electron donor substituents at $\mathrm{C}_{3}$ and $\mathrm{C}_{4}$ preferentially rotate outward in order to maximize the stabilizing interaction with the HOMO of the breaking $\mathrm{C}_{3}-\mathrm{C}_{4}$ bond and to minimize the repulsive interaction with the LUMO of the same bond. Electron acceptor substituents undergo the opposite effect, and, consequently, inward rotation is preferred. Since only certain orbitals are included in the model, a wrong selection of the interacting orbitals leads to wrong predictions. This disadvantage is common for all theories based on a selected group of orbitals, such as the frontier orbital theory. [98] To avoid this flaw, Ponec decided to reinvestigate the problem in terms of an electron density based indicator, such as the molecular similarity approach. [99] He showed that the origin of the torquoselectivity comes from the low electron reorganization required to convert reactants into products.
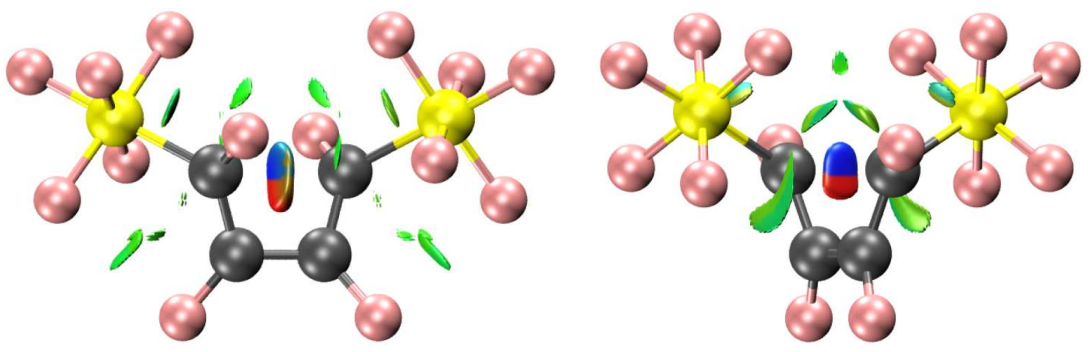

Fig. $17 \mathrm{NCI}$ isosurfaces of $(E, E)$ (left) and $(Z, Z)$ (right) transition states.

Additionally, NCI analyses of both $(Z, Z)$ and $(E, E)$ transition states provide us with topological arguments to understand this differential selectivity. As seen in Figure 17, out of the breaking carbon-carbon covalent interaction (blue isosurface) and its repuslive counterpart ring tension (red isosurface), we can differentiate three types of noncovalent interactions (green isosurfaces):

Type 1 Fluor-fluor interaction between pentafluorosulfanyl groups

Type 2 Pentafluorosulfanyl-carbon interaction

Type 3 Fluor-fluor interaction between pentafluorosulfanyl and fluoro groups

All of them are present in the $(Z, Z)$ transition state, whereas only interactions of type 3 are found in the $(E, E)$ one (see Figure 18). Thus, dispersion interactions between pentafluorosulfanyl groups and those with the carbon cycle should be the driving force of the process. Thus, torquoselectivity can also be understood in terms of secondary interactions as revealed by NCI: within this approach products are driven by the accumulation of noncovalent interactions in the transition state. 


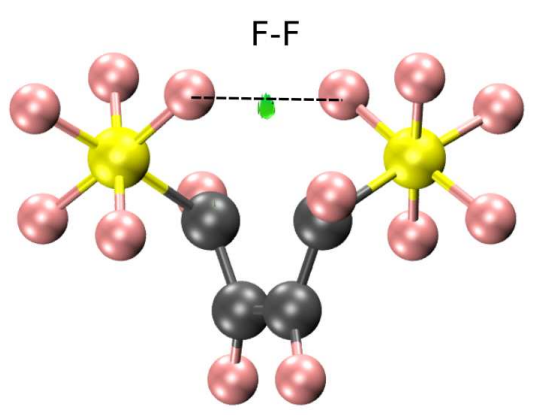

Type 1

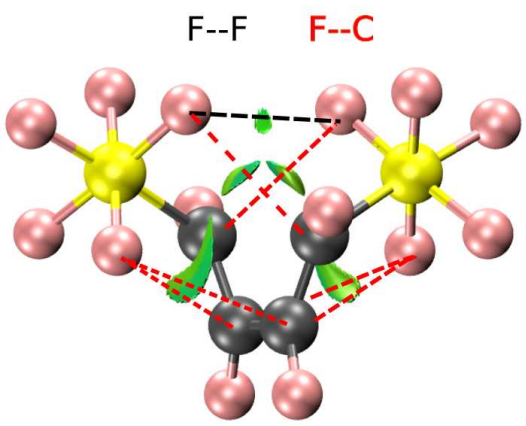

Type $1+2$

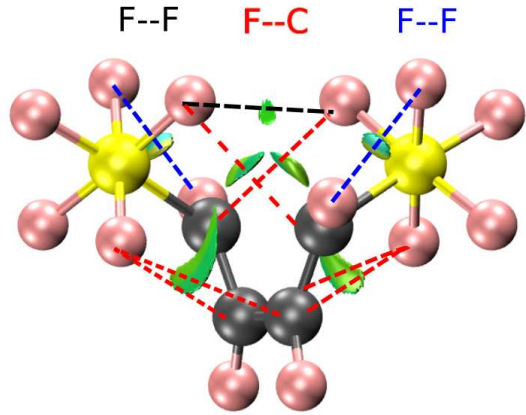

Type $1+2+3$

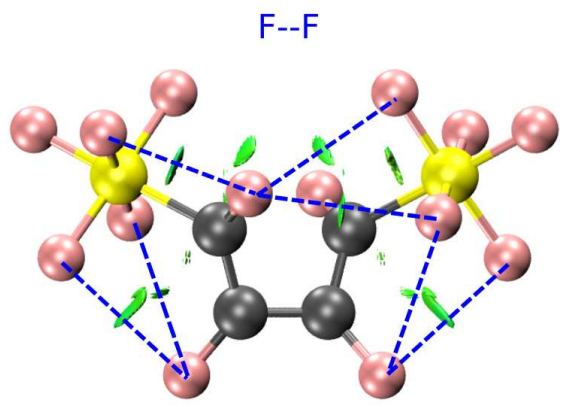

Type 3

Fig. 18 Noncovalent interactions types in $(Z, Z)$ (top and bottom left) and (E,E) (bottom, right) transition states. Black, red and blue dashed lines represent type 1, type 2 and type 3 interactions respectively. 


\section{Summary and conclusions}

In conclusion, non-covalent interactions have a unique signature and their presence can be revealed solely from the electron density. Noncovalent interactions are highly nonlocal and manifest in real space thanks to the NCI analysis: in other words, as low-gradient isosurfaces with low densities. The sign of the second Hessian eigenvalue is used to identify the interaction type, and its strength can be derived from the density on the non-covalent interaction surface.

NCI provides a rapid and rich representation of van der Waals interactions, hydrogen bonds, and steric clashes. If obliged by the size of the system, NCI can be approximated from promolecular densities, so that it only requires the atomic coordinates as input. Thus, it is applicable to large systems, such as proteins or DNA.

Since it is based on the electron density, it is applicable to all types of chemical bonds. We have reviewed here several such examples along the periodic table: halogen bonds, pnicogenic bonds, di-hydrogen bonds. We have even gone to higher densities to show that NCI is also able to reveal and characterize interactions in organometallic systems. Finally, we have looked at the change of chemical interactions along a reaction path, reformulating orbital rules in the torquoselectivity.

In summary, we have shown that the electron density and its derivatives contain all the information needed to characterize all chemical bonds and their change, making NCI a holistic tool in the analysis of weak (and not so weak) interactions. 
Acknowledgements This work undertaken(partially) in the framework of CALSIMLAB is supported by the public grant ANR-11-LABX-0037-01 overseen by the French National Research Agency (ANR) as part of the "Investissements d'Avenir" program (reference: ANR-11-IDEX0004-02) 

Part I

Appendix 



\section{Appendix}

\section{Behavior of Model densities}

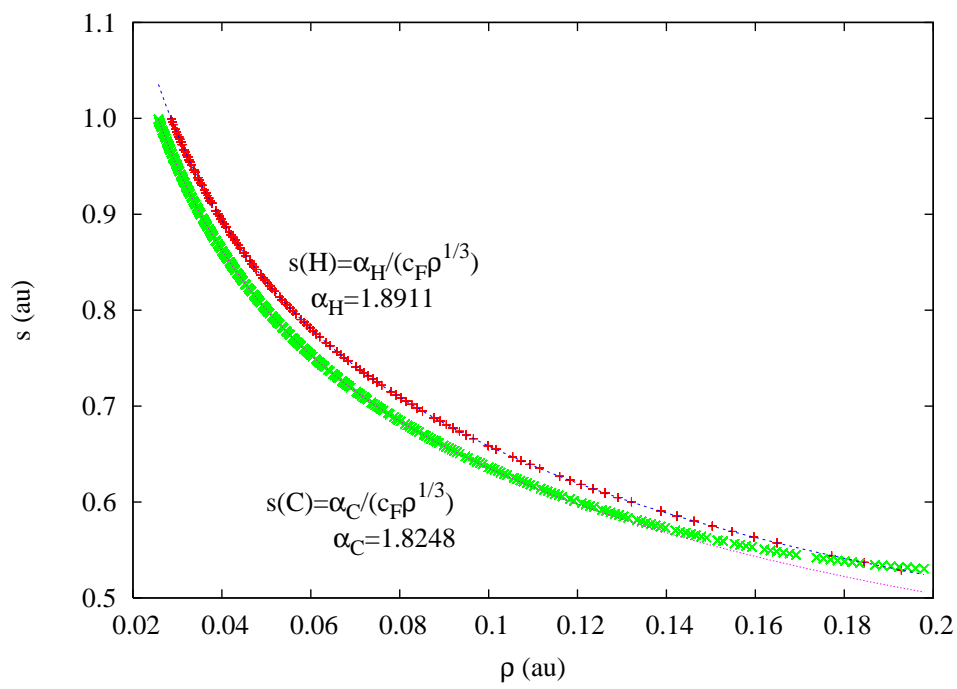

Fig. 19 Behavior of $s(\rho)$ for model densities $\rho=e^{-\alpha r}$ for hydrogen and carbon. 


\section{Parameters for promolecular calculations}

Table 2 Parameterized exponents $\left(\eta_{i}\right)$ and coefficients $\left(c_{i}\right)$ of sphericalized atomic densities, in atomic units.

\begin{tabular}{l|cc|cc|cc} 
Atom & $c_{1}$ & $\eta_{1}$ & $c_{2}$ & $\eta_{2}$ & $c_{3}$ & $\eta_{3}$ \\
\hline \hline $\mathrm{H}$ & 0.2815 & 0.5288 & - & - & - & - \\
$\mathrm{He}$ & 2.437 & 0.3379 & - & - & - & - \\
$\mathrm{Li}$ & 11.84 & 0.1912 & 0.06332 & 0.9992 & - & - \\
$\mathrm{Be}$ & 31.34 & 0.1390 & 0.3694 & 0.6945 & - & - \\
$\mathrm{B}$ & 67.82 & 0.1059 & 0.8527 & 0.5300 & - & - \\
$\mathrm{C}$ & 120.2 & 0.0884 & 1.172 & 0.5480 & - & - \\
$\mathrm{N}$ & 190.9 & 0.0767 & 2.247 & 0.4532 & - & - \\
$\mathrm{O}$ & 289.5 & 0.0669 & 2.879 & 0.3974 & - & - \\
$\mathrm{F}$ & 406.3 & 0.0608 & 3.049 & 0.3994 & - & - \\
$\mathrm{Ne}$ & 561.3 & 0.0549 & 6.984 & 0.3447 & - & - \\
$\mathrm{Na}$ & 760.8 & 0.0496 & 22.42 & 0.2511 & 0.06358 & 1.0236 \\
$\mathrm{Mg}$ & 1016 & 0.0449 & 37.17 & 0.2150 & 0.3331 & 0.7753 \\
$\mathrm{Al}$ & 1319 & 0.0411 & 57.95 & 0.1874 & 0.8878 & 0.5962 \\
$\mathrm{Si}$ & 1658 & 0.0382 & 87.16 & 0.1654 & 0.7888 & 0.6995 \\
$\mathrm{P}$ & 2042 & 0.0358 & 115.7 & 0.1509 & 1.465 & 0.5851 \\
$\mathrm{~S}$ & 2501 & 0.0335 & 158.0 & 0.1369 & 2.170 & 0.5149 \\
$\mathrm{Cl}$ & 3024 & 0.0315 & 205.5 & 0.1259 & 3.369 & 0.4974 \\
$\mathrm{Ar}$ & 3625 & 0.0296 & 260.0 & 0.1168 & 5.211 & 0.4412
\end{tabular}




\section{References}

1. L. Pauling. 1947. General Chemistry, Dover Publications, Inc.

2. J. K. Burdett. 1997. Chemical Bonds-A Dialog. Wiley: Chichester, England.

3. G. Frenking, and S. Shaik (editors). 2007. Special Issue: 90 Years of Chemical Bonding. Journal of Computational Chemistry 28:1-466.

4. G. Frenking, and A. Krapp. 2007. Unicorns in the world of chemical bonding models. Journal of Computational Chemistry 28:15-24.

5. S. Shaik, H. S. Rzepa, and R. Hoffmann. 2013. One molecule, two atoms, three views, four bonds? Angewandte Chemie International Edition 52:3020-33.

6. G. Frenking, and M. Hermann. 2013 Angewandte Chemie International Edition 52:5922-25.

7. C. A. Coulson. 1953. The Spirit of Applied Mathematics, Clarendon Press 20-21.

8. H. Jacobsen. 2010. Kinetic energy density and covalent bonding - a complementary analysis at the border of bond and no bond. Dalton Transactions 39:5426-28.

9. R. G. Parr, P. W. Ayers, and R. F. Nalewajski. 2005. What is an atom in a molecule? The Journal of Physical Chemistry A 109:3957-59.

10. H. Jacobsen. 2013. Topology maps of bond descriptors based on the kinetic energy density and the essence of chemical bonding. Physical Chemistry Chemical Physics 15:5057-66.

11. P. v. R. Schleyer. 2005. Introduction: Delocalization Pi and Sigma. Chemical Reviews 105:3433-35.

12. P. L. A. Popelier. 2007. Preface. Faraday Discussions 135:1-3.

13. I. V. Alabugin, K. M. Gilmore, and P. W. Peterson, P. W. 2011. Hyperconjugation. Wiley Interdisciplinary Reviews: Computational Molecular Science 1:109141.

14. J. F. Gonthier, S. N. Steinmann, M. D. Wodrich, and C. Corminboeuf. 2012. Quantification of "fuzzy" chemical concepts: a computational perspective. Chemical Society Reviews 41:467187.

15. A. Whitaker. Einstein, Bohr and the Quantum Dilemma. 1996. Cambridge. Cambridge University Press.

16. S. Alvarez, R. Hoffmann, and C. Mealli. 2009. A bonding quandary - or - a demonstration of the fact that scientists are not born with logic. Chemistry-A European Journal 15:8358-73.

17. D. Danovich, P. C. Hiberty, W. Wu, H. S. Rzepa, and S. Shaik. 2014. The Nature of the Fourth Bond in the Ground State of C-2 : The Quadruple Bond Conundrum. Chemistry-A European Journal 20:6214-20.

18. P. L. Ayers, R. J. Boyd et al. 2015. Six questions on topology in theoretical chemistry. Computational and Theoretical Chemistry 1053: 2-16.

19. G. N. Lewis. 1916. The atom and the molecule J. Am. Chem. Soc. 38:762-786.

20. K. Fukui, T. Yonezawa, and H. Shingu. 1952. A Molecular Orbital Theory of Reactivity in Aromatic Hydrocarbons. Journal of Chemical Physics 20:722-725.

21. R. B. Woodward, and R. Hoffmann. 1969. The Conservation of Orbital Symmetry Angew. Chem., Int. Ed. Engl. 8:781-932.

22. R. G. Parr, R. A. Donnelly, M. Levy, and W. E. Palke. 1978. Electronegativity: The density functional viewpoint Journal of Chemical Physics 68:3801.

23. R. F. W. Bader. 1991. A quantum theory of molecular structure and its applications. Chem. Rev. 91:893-928.

24. R. F. W. Bader. Atoms in Molecules: A Quantum Theory. 1990. Oxford. International Series of Monographs on Chemistry 22; Oxford Science Publications.

25. C. F. Matta, and R. J. Boyd. In The Quantum Theory of Atoms in Molecules. 2007. New York. Wiley-VCH. 1-34.

26. A. D. Becke, and K. E. J. Edgecombe. 1990. A simple measure of electron localization in atomic and molecular systems. J. Chem. Phys. 92: 5397-5403.

27. B. Silvi, and A. Savin. 1994. Classification of chemical bonds based on topological analysis of electron localization functions. Nature 371:683-686.

28. B. Honig, and A. Nicholls. 1995. Classical electrostatics in biology and chemistry. Science 268:1144-1149. 
29. H. Fenniri, M. Packiarajan, K. L. Vidale, D. M. Sherman, K. Hallenga, K. V. Wood, J. G. Stowell. 2001. Helical Rosette Nanotubes: Design, Self-Assembly, and Characterization. $J$. Am. Chem. Soc. 123:3854-55.

30. P. Kruse, E. R. Johnson, G. A. DiLabio, R. A. Wolkow. 2002. Patterning of Vinylferrocene on $\mathrm{H}-\mathrm{Si}(100)$ via Self-Directed Growth of Molecular Lines and STM-Induced Decomposition. Nano Lett. 2:807-810.

31. S. S. Sheiko, F. C. Sun, A. Randall, D. Shirvanyants, M. Rubinstein, H. Lee, K. Matyjaszewski. 2006. Adsorption-induced scission of carbon-carbon bonds. Nature. 440:191-194.

32. G. A. DiLabio, P. G. Piva, P. Kruse, R. A. Wolkow. 2004. Dispersion interactions enable the self-directed growth of linear alkane nanostructures covalently bound to silicon. J. Am. Chem. Soc. 126:16048-50.

33. P. A. Kollman. 1977. Noncovalent interactions Chem. Rev. 10:365-371.

34. J. Cerniý, P. Hobza. 2007. Non-covalent interactions in biomacromolecules. Phys. Chem. Chem. Phys. 9:5291-5303.

35. A. L. Lehninger, D. L. Nelson, M. M. Cox. 1993. Principles of Biochemistry. Second edition. Worth Publishers, Inc.

36. N. Krishnamoorthy, M. H. Yacoub, S. N. Yaliraki. 2011. A computational modeling approach for enhancing self-assembly and biofunctionalisation of collagen biomimetic peptides. Biomaterials 32:7275-85.

37. A. Dutta, A. D. Jana, S. Gangopadhyay, K. Kumar Das, J. Marek, R. Marek, J. Brus, M. Ali. 2011. Unprecedented $\pi \pi$ interaction between an aromatic ring and a pseudo-aromatic ring formed through intramolecular H-bonding in a bidentate Schiff base ligand: crystal structure and DFT calculations. Phys. Chem. Chem. Phys. 13:15845-53.

38. A. Gavezzotti. Molecular Aggregation. Structure Analysis and Molecular Simulation of Crystals and Liquids. 2007. Oxford. IUCr monographs on Crystallography n. 19, Oxford University Press.

39. S. Keinan, M. A. Ratner, T. J. Marks. 2004. Molecular zippers-designing a supramolecular system. J. Chem. Phys. Lett. 392:291-296.

40. G. R. Desiraju. Crystal Engeneering. The design of organic Solids 1989. Amsterdam. Elsevier

41. G. M. Day et al. 2009. Acta Cryst. B. 65:107-125.

42. J. M. Word, S. C. Lovell, T. H. LaBean, H. C. Taylor, M. E. Zalis, B. K. Presley, J. S. Richardson, and D. C. Richardson. 1999. Asparagine and glutamine: using hydrogen atom contacts in the choice of side-chain amide orientation. J. Mol. Biol. 285:1735-1747.

43. I. W. Davis, A. Leaver-Fay, V. B. Chen, J. N. Block, G. J. Kapral, X. Wang, L. W. Murray, W. B. Arendall III, J. Snoeyink, J. S. Richardson, and D. C. Richardson. 2007. MolProbity: all-atom contacts and structure validation for proteins and nucleic acids. Nucleic Acids Res. 35:W375-W383.

44. V. Sobolev, A. Sorokine, J. Prilusky, E. E. Abola, and M. Edelman. 1999. Automated analysis of interatomic contacts in proteins. Bioinformatics 15:327-332.

45. I. K. McDonald, and J. M. Thornton. 1994. Satisfying hydrogen bonding potential in proteins. J. Mol. Biol. 238:777-793.

46. M.E. Alikhani, F. Fuster, and B. Silvi. 2005. What can tell the topological analysis of ELF on hydrogen bonding? Struct. Chem. 16:203-210.

47. R. D. Cramer III, D. E. Patterson, and J. D. Bunce. 1988. Comparative molecular field analysis (CoMFA). 1. Effect of shape on binding of steroids to carrier proteins. J. Am. Chem. Soc. 110:5959-5967.

48. J. Pilmé, J.-P. Piquemal. 2008. Advancing beyond charge analysis using the electronic localization function: Chemically intuitive distribution of electrostatic moments J. Comput. Chem. 29:1440-49.

49. P. Hohenberg, and W. Kohn. 1964. Inhomogeneous electron gas Phys. Rev. B 136:B864.

50. E. R. Johnson, S. Keinan, P. Mori-Sanchez, J. Contreras-García, A. J. Cohen, and W. Yang. 2010. Revealing Noncovalent Interactions J. Am. Chem. Soc. 132:6498-6506.

51. J. Contreras-García, E. Johnson, S. Keinan, R. Chaudret, J.-P. Piquemal, D. Beratan, and W. Yang. 2011. NCIPLOT: a program for plotting non-covalent interaction regions J. Chem. Theor. Comp. 7:625-632. 
52. J. Contreras-García, E. R. Johnson, W. Yang. 2011. Analysis of Hydrogen-Bond Interaction Potentials from the Electron Density: Integration of Noncovalent Interaction Regions J. Phys. Chem. A 115:12983-12990.

53. A. D. Becke. 1995. Modern Electronic Structure Theory. World Scientific, Yarkony.

54. A. J. Cohen, P. Mori-Sánchez, and W. Yang. 2008. Insights into current limitations of density functional theory. Science 321:792-794.

55. A. Zupan, K. Burke, M. Ernzerhof, , J. P. Perdew. 1997. Distributions and averages of electron density parameters: Explaining the effects of gradient corrections. J. Chem. Phys. 106:10184193.

56. A.D. Becke. 1993. Density-functional thermochemistry. III. The role of exact exchange. $J$. Chem. Phys. 98:5648-52.

57. C. Lee, W. Yang, and R.G. Parr. 1988. Development of the Colle-Salvetti correlation-energy formula into a functional of the electron density. Phys. Rev. B. 37:785.

58. G. Arfken, G. Mathematical Methods for Physicists. 1985. Orlando Academic Press.

59. R. F. W. Bader, H. Essén. 1984. The characterization of atomic interactions. J. Chem. Phys. 80:1943-1960.

60. J. R. Lane, J. Contreras-García, J.-P. Piquemal, B. J. Miller, and H. G. Kjaergaard. 2013. Are Bond Critical Points Really Critical for Hydrogen Bonding? J. Chem. Theory Comp. 9:32633266.

61. J. Contreras-García, M. Calatayud, J.-P. Piquemal, and J. M. Recio. 2012. Ionic interactions: Comparative topological approach Comp. Theo. Chem. 998:193-201.

62. R. Chaudret, B. de Courcy, J. Contreras-García, E. Gloaguen, A. Zehnacker-Rentien, M. Mons, and J.-P. Piquemal. 2013. Unraveling Non Covalent Interactions within Flexible Biomolecules: from electron density topology to gas phase spectroscopy Phys. Chem. Chem. Phys. 16:9876-9891.

63. M. A. Spackman, E. N. Maslen. 1986. Chemical properties from the promolecule. J. Phys. Chem. 90:2020-2027.

64. A. Martin Pendás, V. Luaña, L. Pueyo, E. Francisco, P. Mori-Sánchez. 2002. Hirshfeld surfaces as approximations to interatomic surfaces. J. Chem. Phys. 117:1017-1023.

65. S. Fiedler, J. Broecker, , S. Keller. 2010. Protein folding in membranes. Cellular and Molecular Life Sciences, 67:1779-98.

66. K. A. Dill. 1990. Dominant forces in protein folding. Biochemistry, 29:7133-55.

67. B. J. Deppmeier, A. J. Driessen, W. Hehre, J. A. Johnson, P. E. Klunzinger, and M. Watanabe. 2002. Spartan ES 1.0.2. Wavefunction Inc., Irvine.

68. X.-J. Lu , and W. K. Olson. 3DNA: a software package for the analysis, rebuilding and visualization of three-dimensional nucleic acid structures. Nucleic Acids Res. 31:5108-121.

69. http://rutchem.rutgers.edu/ olson/Tsukuba/.

70. E. Espinosa, E. Molins, , C. Lecomte. 1988. Hydrogen bond strengths revealed by topological analyses of experimentally observed electron densities. Chem. Phys. Lett. 285:170-173.

71. R. Wieczorek, J. J. Dannenberg. 2004. Comparison of fully optimized $\alpha$-and 310-helices with extended $\beta$-strands. An oniom density functional theory study. J. Am. Chem. Soc. 126:14198205.

72. R. Viswanathan, A. Asensio, and J. J. Dannenberg. 2004. Cooperative hydrogen-bonding in models of antiparallel $\beta$-sheets. J. Phys. Chem. A. 108:9205-212.

73. P. Jurecka , and P. Hobza. 2003. True Stabilization Energies for the Optimal Planar HydrogenBonded and Stacked Structures of Guanine -..Cytosine, Adenine -..Thymine, and Their 9-and 1-Methyl Derivatives: Complete Basis Set Calculations at the MP2 and CCSD (T) Levels and Comparison with Experiment. J. Am. Chem. Soc. 125:15608-613.

74. E. Arunan, G. R. Desiraju, R. A. Klein, J. Sadlej, S. Scheiner, I. Alkorta, D. C. Clary, R. H. Crabtree, J. J. Dannenberg, P. Hobza, H. G. Kjaergaard, A. C. Legon, B. Mennucci and D. J. Nesbitt. 2011. Definition of the hydrogen bond (IUPAC Recommendations 2011). Pure Appl. Chem. 83:1637-1641.

75. P. Metrangolo and G. Resnati. 2001. Halogen bonding: a paradigm in supramolecular chemistry. Chemistry - A European Journal 7:2511-2519. 
76. D. Manna and G. Mugesh. 2012 Regioselective deiodination of thyroxine by iodothyronine deiodinase mimics: an unusual mechanistic pathway involving cooperative chalcogen and halogen bonding. J. Am. Chem. Soc. 134:4269-4279.

77. S. Scheiner. A New Noncovalent Force: Comparison of P $\cdots$ N Interaction with Hydrogen and Halogen Bonds J. Chem. Phys. 134:094315-094319.

78. D. Mani and E. Arunan. 2013. The X-C $\cdots \mathrm{Y}(\mathrm{X}=\mathrm{O} / \mathrm{F}, \mathrm{Y}=\mathrm{O} / \mathrm{S} / \mathrm{F} / \mathrm{Cl} / \mathrm{Br} / \mathrm{N} / \mathrm{P})$ 'Carbon Bond' and Hydrophobic Interactions. Phys. Chem. Chem. Phys. 15:14377-14383.

79. H. Matter, M. Nazaré, S. Güssregen, D. W. Will, H. Schreuder, A. Bauer, M. Urmann, K. Ritter, M. Wagner, and V. Wehner. 2009. Evidence for C-Cl/C-Br $\pi$ interactions as an important contribution to protein-ligand binding affinity. Angewandte Chemie, 121:2955-2960.

80. L. A. Hardegger, B. Kuhn, B. Spinnler, L. Anselm, R. Ecabert, M. Stihle, B. Gsell, R. Thoma, J. Diez, J. Benz, et al. 2011. Systematic investigation of halogen bonding in protein-ligand interactions. Angewandte Chemie International Edition 50:314-318.

81. K. A. Peterson, B. C. Shepler, D. Figgen, and H. Stoll. 2006. On the spectroscopic and thermochemical properties of $\mathrm{ClO}, \mathrm{BrO}, \mathrm{IO}$, and their anions. The Journal of Physical Chemistry A 110:13877-13883.

82. J. Murray, P. Lane and P. Politzer. 2007. A Predicted New Type of Directional Noncovalent Interaction. Int. J. Quantum Chem. 107:2286-2292.

83. J. George, V. L. Deringer and R. Dronskowski. Cooperativity of Halogen, Chalcogen, and Pnictogen Bonds in Infinite Molecular Chains by Electronic Structure Theory. J. Phys. Chem. A 118:3193-3200.

84. A.C. Legon, B.P. Roberts, and A.L. Wallwork. 1990. Rotational spectra and geometries of the gas-phase dimers $\left(\mathrm{CH}_{4}, \mathrm{HF}\right)$ and $\left(\mathrm{CH}_{4}, \mathrm{HCl}\right)$ Chem. Phys. Lett. 173:107-114

85. W. J. Stevens, W. H. Fink. 1987. Frozen fragment reduced variational space analysis of hydrogen bonding interactions. Application to the water dimer. Chem. Phys. Lett., 139:15-22.

86. P. L. A. Popelier. 1998. Characterization of a dihydrogen bond on the basis of the electron density. J. Phys. Chem. A, 102:1873-78.

87. C. A. Morrison, M. M. Siddick. 2004. Dihydrogen bonds in solid $\mathrm{BH}_{3} \mathrm{NH}_{3}$. Angew. Chem. Int. Ed., 116:4780-4782.

88. C. F. Matta, J. Hernndez-Trujillo, T. Tang, and R. F. W. Bader. 2003. Hydrogen-hydrogen bonding: a stabilizing interaction in molecules and crystals. Chem. Eur. J., 9:1940-1951.

89. Scienomics; MAPS platform, version 3.4, 2014, France

90. M. Valiev, E.J. Bylaska, N. Govind, K. Kowalski, T.P. Straatsma, H.J.J. van Dam, D. Wang, J. Nieplocha, E. Apra, T.L. Windus, and W.A. de Jong. 2010. NWChem: a comprehensive and scalable open-source solution for large scale molecular simulations. Comput. Phys. Commun. 181:1477-89.

91. T. B. Richardson, de Gala S., Crabtree, , P. E. M. R. H.; Siegbahn. 1995. Unconventional

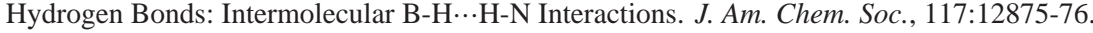

92. E. Matito, J. Poater, M. Duran, and M. Solà. 2006. Electron Fluctuation in Pericyclic and Pseudopericiclyc Reations. A European Journal of Chemical Physics and Physical Chemistry 7:111-13.

93. N. Gillet, R. Chaudret, J. Contreras-Garcia, W. Yang, B. Silvi, and J.-P. Piquemal. 2012.Coupling Quantum Interpretative Techniques: Another Look at Chemical Mechanism in Organic Reactions. Journal of Chemical Theory and Computation 8:3993-97.

94. R. B. Woodward, R. J. Hoffmann. 1965. Selection Rules for Concerted Cycloaddition Reactions. Journal of the American Chemical Society 87:2046-48.

95. K. N. Houk, Y. Li, and J. D. Evanseck. 1992. Transition Structures of Hydrocarbon Pericyclic Reactions. Angewandte Chimie International Edition 31:682-708

96. W. Kirmse, N. G. Rondan, and K. N. Houk. 1984. Stereoselective substituent effects on conrotatory electrocyclic reaction of cyclobutenes. Journal of the American Chemical Society 106:7989-91-

97. N. G. Rondan, K. N. Houk. 1984. Theory of setereoselection in conrotatory electrocyclic reactions of substitued cyclobutenes. Journal of the American Chemical Society 107:2099111 
98. M. J. S. Deward. 1989. A Critique of Frontier Orbital Theory. Journal of Molecular Structure (Theochem) 200:301-23.

99. R. Ponec, G. Yuzhakov, and J. Pecka. 1996. Similarity approach to chemical reactivity. Torquoselectivity in pericyclic reactions. Journal of Mathematical Chemistry 20:301-10.

100. B. De Courcy, J.-P. Dognon, C. Clavaguéra, N. Gresh, J.-P. Piquemal. 2011. Interactions within the Alcohol Dehydrogenase (ADH) Zn(II)-metalloenzyme active site: interplay between subvalence, electron correlation/dispersion and charge transfer/induction effects. Int. J. Quantum Chem. 111:1213-1221.

101. Y.-M. Lee, C. Lim. 2011. Factors controlling the reactivity of zinc finger cores. J. Am. Chem. Soc. 133:8691-8703. 